\title{
SOME INTEGRAL INEQUALITIES WITH APPLICATIONS TO THE IMBEDDING OF SOBOLEV SPACES DEFINED OVER IRREGULAR DOMAINS
}

\author{
BY
}

\author{
R. A. $\operatorname{ADAMS}(1)$
}

ABSTRACT. This paper examines the possibility of extending the Sobolev Imbedding Theorem to certain classes of domains which fail to have the "cone property" normally required for that theorem. It is shown that no extension is possible for certain types of domains (e.g. those with exponentially sharp cusps or which are unbounded and have finite volume), while extensions are obtained for other types (domains with less sharp cusps). These results are developed via certain integral inequalities which generalize inequalities due to Hardy and to Sobolev, and are of some interest in their own right.

The paper is divided into two parts. Part I establishes the integral inequalities; Part II deals with extensions of the imbedding theorem. Further introductory information may be found in the first section of each part.

\section{PART I. INTEGRAL INEQUALITIES}

1.1 Introduction. The inequalities developed in this section generalize certain well-known integral inequalities of G. H. Hardy and S. L. Sobolev and concern estimates for weighted $L^{q}$-norms, uniform norms and Hölder norms for continuously differentiable functions defined on open intervals, cones or balls in terms of weighted $L^{p}$-norms of the function and its first derivatives. The inequalities will be used in Part II to prove imbedding theorems for (unweighted) Sobolev spaces defined over irregular domains.

The one-dimensional case is treated in $\$ 1.2$, and the results obtained extended to $(n+1)$-dimensional Euclidean space $E_{n+1}$ in the remaining sections, $\S 1.3$ dealing with bounds for weighted $L^{q}$-norms, and $\$ 1.4$ with pointwise bounds and Hölder conditions.

Functions $u$ may be assumed complex-valued in general. We shall not be concerned with the problem of finding the best constants for our inequalities.

1.2 The one-dimensional case. Throughout this section we consider functions $u$ continuously differentiable on an open interval $(0, T)$ for fixed $T>0$. In each inequality studied it may be assumed that the right-hand side is finite.

Received by the editors May 31, 1972.

AMS (MOS) subject classifications (1970). Primary 46E35, 26A84; Secondary 26A87.

Key words and phrases. Sobolev space, imbedding theorem, integral inequality.

(1) Research partially supported by the National Research Council of Canada under Operat ing Grant number A-3973. 
1.2.1 Lemma. If $\delta \geq 1$ and $\alpha>0$ then

$$
\int_{0}^{T}|u(t)|^{\delta} t^{\alpha-1} d t \leq \frac{\alpha+1}{\alpha T} \int_{0}^{1}|u(t)|^{\delta} t^{\alpha} d t+\frac{2 \delta}{\alpha} \int_{0}^{T}|u(t)|^{\delta-1}\left|u^{\prime}(t)\right| t^{a} d t .
$$

Proof. It is sufficient to prove (1) for $\delta=1$. Integration by parts yields

$$
\int_{0}^{T}|u(t)|\left[a t^{a-1}-\frac{a+1}{T} t^{a}\right] d t=-\int_{0}^{T}\left[t^{a}-\frac{1}{T} t^{a+1}\right] \frac{d}{d t}|u(t)| d t .
$$

Transposition and estimation of the term on the right gives

$$
a \int_{0}^{T}|u(t)| t^{a-1} d t \leq \frac{a+1}{T} \int_{0}^{T}|u(t)| t^{a} d t+2 \int_{0}^{T}\left|u^{\prime}(t)\right| t^{a} d t
$$

which is the desired result.

1.2.2 Theorem. If $p \geq 1$ and $\alpha>p-1$ then

$$
\int_{0}^{T}|u(t)|^{p} t^{\alpha-p} d t \leq 2^{p-1}\left[\frac{\alpha+2+p}{\alpha+1-p}\right]^{p} \int_{0}^{T}\left(\frac{|u(t)|^{p}}{T^{p}}+\left|u^{\prime}(t)\right|^{p}\right) t^{\alpha} d t .
$$

Proof. Taking $\delta=p$ and replacing $a$ by $\alpha-p+1$ in (1), and then using Hölder's inequality, we obtain

$$
\begin{aligned}
\int_{0}^{T} & |u(t)|^{p} t^{a-p} d t \\
& \leq \frac{a+2-p}{(a+1-p) T} \int_{0}^{T}|u(t)|^{p} t^{a-p+1} d t+\frac{2 p}{a+1-p} \int_{0}^{T}|u(t)|^{p-1}\left|u^{\prime}(t)\right| t^{a-p+1} d t \\
& \leq \frac{a+2+p}{a+1-p}\left\{\int_{0}^{T}|u(t)|^{p} t^{a-p} d t\right\}^{(p-1) / p}\left\{\int_{0}^{T}\left(\frac{|u(t)|}{T}+\left|u^{\prime}(t)\right|\right)^{p} t^{a} d t\right\}^{1 / p}
\end{aligned}
$$

from which follows

$$
\left\{\int_{0}^{T}|u(t)|^{p} t^{a-p} d t\right\}^{1 / p} \leq \frac{\alpha+2+p}{\alpha+1-p}\left\{2^{p-1} \int_{0}^{T}\left(\frac{|u(t)|^{p}}{T^{p}}+\left|u^{\prime}(t)\right|^{p}\right) t^{\alpha} d t\right\}^{1 / p}
$$

whence the inequality (2).

Remark. If $t^{a / p} u(t)$ belongs to $L^{p}(0, \infty)$ then letting $T \rightarrow \infty$ in (2) we obtain

$$
\int_{0}^{\infty}|u(t)|^{p} t^{a-p} d t \leq \text { const } \int_{0}^{\infty}\left|u^{\prime}(t)\right|^{p} t^{a} d t
$$

This resembles the classical Hardy inequality (e.g. see Zygmund [14, p. 20]), which is usually proved for $a<p-1$ and functions $u$ such that $u(t) \rightarrow 0$ as $t \rightarrow 0+$, though it also known for $a>p-1$ if $u(t) \rightarrow 0$ as $t \rightarrow \infty$. Generalization of Hardy's inequality to finite intervals usually involves boundary conditions on the function $u$ at one or another of the endpoints but (2) above replaces such 
boundary restrictions by the requirement that $\int_{0}^{T}|u(t)|^{p} t^{a} d t$ be included on the right-hand side.

1.2.3 Lemma. If $\delta \geq 1$ and $\alpha>0$ we have the following pair of inequalities:

$$
\begin{aligned}
& \sup _{0<t<T}|u(t)|^{\delta} \leq \frac{2}{T} \int_{0}^{T}|u(t)|^{\delta} d t+\delta \int_{0}^{T}|u(t)|^{\delta-1}\left|u^{\prime}(t)\right| d t \\
& \sup _{0<t<T}|u(t)|^{\delta} t^{\alpha} \leq \frac{\alpha+3}{T} \int_{0}^{T}|u(t)|^{\delta} t^{\alpha} d t+3 \delta \int_{0}^{T}|u(t)|^{\delta-1}\left|u^{\prime}(t)\right| t^{a} d t .
\end{aligned}
$$

Proof. Again we need only establish the inequalities for the case $\delta=1$. If $0<t \leq T / 2$ we obtain by integration by parts

$$
\int_{0}^{T / 2}\left|u\left(t+\frac{T}{2}-\tau\right)\right| d \tau=\frac{T}{2}|u(t)|-\int_{0}^{T / 2} \tau \frac{d}{d \tau}\left|u\left(t+\frac{T}{2}-\tau\right)\right| d \tau
$$

whence $|u(t)| \leq(2 / T) \int_{0}^{T}|u(\sigma)| d \sigma+\int_{0}^{T}\left|u^{\prime}(\sigma)\right| d \sigma$. For $T / 2 \leq t<T$ the same inequality follows partial integration of the integral $\int_{0}^{T / 2}|u(t+\tau-T / 2)| d \tau$. This proves (3).

Replacing $u(t)$ by $u(t) t^{a}$ in (3) (with $\delta=1$ ) we obtain

$$
\begin{aligned}
\sup _{0<t<T}|u(t)| t^{\alpha} \leq & \frac{2}{T} \int_{0}^{T}|u(t)| t^{\alpha} d t+\int_{0}^{T}\left[\left|u^{\prime}(t)\right| t^{\alpha}+\alpha|u(t)| t^{\alpha-1}\right] d t \\
\leq & \frac{2}{T} \int_{0}^{T}|u(t)| t^{\alpha} d t+\int_{0}^{T}\left|u^{\prime}(t)\right| t^{\alpha} d t \\
& +\alpha\left\{\frac{\alpha+1}{\alpha T} \int_{0}^{T}|u(t)| t^{a} d t+\frac{2}{\alpha} \int_{0}^{T}\left|u^{\prime}(t)\right| t^{\alpha} d t\right\}
\end{aligned}
$$

where Lemma 1.2.1 has been used to get the last inequality. This is the desired result for $\delta=1$.

1.2.4 Theorem. If $p \geq 1$ and $a>p-1$ there exists a constant $K$ depending only on a and pucb that

$$
\left\{\int_{0}^{T}|u(t)|^{\gamma} t^{\alpha} d t\right\}^{1 / \gamma} \leq K\left\{\int_{0}^{T}\left(\frac{|u(t)|^{p}}{T^{p}}+\left|u^{\prime}(t)\right|^{p}\right) t^{\alpha} d t\right\}^{1 / p}
$$

where $\gamma=(\alpha+1) p /(\alpha+1-p)$.

Proof. In this proof the symbols $K_{i}$ denote various constants depending on $\alpha$ and $p$. We have from Lemma 1.2.3 


$$
\begin{aligned}
\sup _{0<t<T}|u(t)|^{p} t^{a+1-p} \leq K_{1} \int_{0}^{T}|u(t)|^{p-1}\left[\frac{|u(t)|}{T}+\left|u^{\prime}(t)\right|\right] t^{\alpha+1-p} d t \\
\quad \leq K_{1}\left\{\int_{0}^{T}|u(t)|^{p} t^{a-p} d t\right\}^{(p-1) / p}\left\{\int_{0}^{T}\left[\frac{|u(t)|}{T}+\left|u^{\prime}(t)\right|\right]^{p} t^{a} d t\right\}^{1 / p} \\
\leq K_{2} \int_{0}^{T}\left[\frac{|u(t)|^{p}}{T^{p}}+\left|u^{\prime}(t)\right|^{p}\right] t^{a} d t
\end{aligned}
$$

where Theorem 1.2.2 has been used in obtaining the last inequality. Since $\gamma-p$ $=p^{2} /(\alpha+1-p)$ we have

$$
\begin{aligned}
\int_{0}^{T}|u(t)|^{\gamma} t^{\alpha} d t & \leq \sup _{0<t<T}\left[|u(t)|^{\gamma-p} t^{p}\right] \int_{0}^{T}|u(t)|^{p} t^{a-p} d t \\
& \leq K_{3}\left\{\int_{0}^{T}\left[\frac{|u(t)|^{p}}{T^{p}}+\left|u^{\prime}(t)\right|^{p}\right] t^{\alpha} d t\right\}^{p /(a+1-p)} \int_{0}^{T}|u(t)|^{p} t^{\alpha-p} d t \\
& \leq K_{4}\left\{\int_{0}^{T}\left[\frac{|u(t)|^{p}}{T^{p}}+\left|u^{\prime}(t)\right|^{p}\right] t^{a} d t\right\}^{(a+1) /(a+1-p)}
\end{aligned}
$$

once again by Theorem 1.2.2. This is the desired result.

Corollary. If $p \geq 1$ and $a>p-1$, and if $0 \leq s \leq p$, then there exists a constant $K_{s}$ depending on $\alpha$ and $p$ such that

$$
\left\{\int_{0}^{T}|u(t)|^{\lambda} t^{\alpha-s} d t\right\}^{1 / \lambda} \leq K_{s}\left\{\int_{0}^{T}\left[\frac{|u(t)|^{p}}{T^{p}}+\left|u^{\prime}(t)\right|^{p}\right] t^{\alpha} d t\right\}^{1 / p}
$$

where $\lambda=(\alpha+1-s) p /(\alpha+1-p)$.

Proof. Clearly $p \leq \lambda \leq \gamma$. Set $q=(\gamma-p) /(\lambda-p), q^{\prime}=(\gamma-p) /(\gamma-\lambda)$. Then $1 / q+1 / q^{\prime}=1$ and we have by Hölder's inequality

$$
\int_{0}^{T}|u(t)|^{\lambda} t^{a-s} d t \leq\left\{\int_{0}^{T}|u(t)|^{\gamma} t^{\alpha} d t\right\}^{1 / q}\left\{\int_{0}^{T}|u(t)|^{p_{t}{ }^{\alpha}-p} d t\right\}^{1 / q^{\prime}}
$$

whence (6) follows from (5) and (2).

We conclude this section with brief consideration of the case $a<p-1$.

1.2.5 Lemma. If $p \geq 1$ and $a<p-1$ then

$$
\sup _{0<t<T}|u(t)|^{p} \leq\left[\frac{2(p-1)}{p-\alpha-1}\right]^{p-1} T^{p-a-1} \int_{0}^{T}\left[\frac{|u(t)|^{p}}{T^{p}}+\left|u^{\prime}(t)\right|^{p}\right] t^{\alpha} d t
$$

Proof. For $0<t, \tau<T$ we have $u(t)-u(\tau)=\int_{\tau}^{t} u^{\prime}(\sigma) d \sigma$ from which 
$|u(t)| \leq|u(\tau)|+\int_{0}^{T}\left|u^{\prime}(\sigma)\right| d \sigma$. Integration of $\tau$ over $(0, T)$ and application of Hölder's inequalitv in case $p>1$ yields

$$
\begin{aligned}
& T|u(t)| \leq \int_{0}^{T}|u(\tau)| d \tau+T \int_{0}^{T}\left|u^{\prime}(\sigma)\right| d \sigma \\
& \quad \leq T\left\{2^{p-1} \int_{0}^{T}\left[\frac{|u(\sigma)|^{p}}{T^{p}}+\left|u^{\prime}(\sigma)\right|^{p}\right] \sigma^{\alpha} d \sigma\right\}^{1 / p}\left\{\int_{0}^{T} \sigma^{-\alpha /(p-1)} d \sigma\right\}^{(p-1) / p}
\end{aligned}
$$

from which (7) follows. If $p=1$, (7) follows from the first inequality above.

Remarks. 1. For $a \leq p-1$ and $T<\infty$ (5) holds for $1 \leq \gamma<\infty$.

2. Under the assumptions of Lemma 1.2.5 it can be shown further that

$$
\sup _{0<t, \tau<T} \frac{|u(t)-u(\tau)|}{|t-\tau|^{\mu}} \leq \text { const }\left\{\int_{0}^{T}\left[\frac{|u(t)|^{p}}{T^{p}}+\left|u^{\prime}(t)\right|^{p}\right] t^{a} d t\right\}^{1 / p}
$$

where $\mu=1-(\alpha+1) / p$. We defer the proof of this inequality as it is similar to, and a special case of, that of Theorem 1.4.3 below.

1.3 The multi-dimensional case $-L^{p}$ estimates. In this section $x=\left(x_{1}, \ldots\right.$, $\left.x_{n+1}\right)$ will denote a point in $(n+1)$-dimensional Euclidean space $E_{n+1}(n \geq 1)$, and we shall use the spherical polar coordinate representation $x=\left(\rho, \phi_{1}, \phi_{2}\right.$, $\left.\cdots, \phi_{n}\right)=(\rho, \phi)$ where $\rho \geq 0,-\pi \leq \phi_{1} \leq \pi, 0 \leq \phi_{2}, \cdots, \phi_{n} \leq \pi$, and

$$
\begin{aligned}
x_{1} & =\rho \sin \phi_{1} \sin \phi_{2} \cdots \sin \phi_{n}, \\
x_{2} & =\rho \cos \phi_{1} \sin \phi_{2} \cdots \sin \phi_{n}, \\
x_{3} & =\rho \cos \phi_{2} \cdots \sin \phi_{n}, \\
\cdot & \\
\cdot & \\
x_{n+1} & =\rho \cos \phi_{n} .
\end{aligned}
$$

The volume element is $d x=d x_{1} d x_{2} \cdots d x_{n+1}=\rho^{n} \Pi_{j=1}^{n} \sin ^{j-1} \phi_{j} d \rho d \phi$ where $d \phi=d \phi_{1} \cdots d \phi_{n}$.

We introduce functions $r_{k}=r_{k}(x), 1 \leq k \leq n+1$, as follows:

$$
\begin{aligned}
r_{1}(x) & =\rho\left|\sin \phi_{1}\right| \prod_{j=2}^{n} \sin \phi_{j}, \\
r_{k}(x) & =\rho \prod_{j=k}^{n} \sin \phi_{j}, \quad k=2,3, \ldots, n, \\
r_{n+1}(x) & =\rho .
\end{aligned}
$$

For $1 \leq k \leq n, r_{k}(x)$ is the distance from $x$ to the coordinate (hyper-) plane 
spanned by the axes $x_{k+1}, \cdots, x_{n+1}$, while ${ }_{n+1}(x)$ is just the distance from $x$ to the origin. In connection with the use of product symbols of the form $P=$ $\Pi_{j=k}^{m} P_{j}$, be it agreed hereafter that $P=1$ if $m<k$.

Throughout this section $\Omega$ shall denote an open, conical domain in $E_{n+1}$ specified in polar coordinates by the inequalities

$$
0<\rho<a, \quad-\beta_{1}<\phi_{1}<\beta_{1}, \quad 0 \leq \phi_{j}<\beta_{j}, \quad j=2,3, \cdots, n,
$$

where $0<\beta_{i} \leq \pi$. (The inequalities " $<$ " are replaced by " $\leq$ " for any $\beta_{i}=\pi$. If all $\beta_{i}=\pi$ then the first inequality is replaced by $0 \leq \rho<a$.)

In the lemmas and theorems that follow it is always assumed that the functions $u$ considered belong to $C^{1}(\Omega)$ (i.e. are continuously differentiable on $\Omega$ ) and that the right-haind sides of the stated inequalities are finite. The constants $K, Q, K{ }_{i}$, $Q_{i}, K^{*}, Q^{*}$ etc. occurring in the statements and proofs may depend on $\alpha, p, n, \beta_{i}$, and $\delta$ but not on $a$ or $u$.

Our first result generalizes Lemma 1.2.1.

1.3.1 Lemma. Let $\delta \geq 1$. Suppose that either $m=k=1$ or $2 \leq m \leq n+1$, $1 \leq k \leq n+1$, and suppose also that $\alpha>1-k$. Then

$$
\int_{\Omega}|u(x)|^{\delta} \frac{\left[r_{k}(x)\right]^{\alpha}}{r_{m}(x)} d x \leq K_{m, k} \int_{\Omega}|u(x)|^{\delta}-1\left[\frac{|u(x)|}{a}+\left|\nabla_{u}(x)\right|\right]\left[r_{k}(x)\right]^{a} d x .
$$

Proof. We assume $\delta=1$; it is clearly sufficient to prove (3) for this case. Write $\Omega=\Omega_{+} \cup \Omega_{-}$where $\Omega_{+}$consists of those points of $\Omega$ for which $\phi_{1} \geq 0$. We shall actually prove (3) for $\Omega_{+}$(which, however, we shall continue to call $\Omega$ ). A similar proof holds for $\Omega$ _ and hence (3) holds for the given $\Omega$. Accordingly, assume $\Omega=\Omega_{+}$. For $k \leq m$ we may write (3) in the form

$$
\int_{\Omega}|u| \prod_{j=1}^{k-1} \sin ^{j-1} \phi_{j} \prod_{j=k}^{m-1} \sin ^{a_{+j-1}} \phi_{j} \prod_{j=m}^{n} \sin ^{a_{+j-2}} \phi_{j} \rho^{a_{+n-1}} d \rho d \phi
$$

$$
\leq K_{m, k} \int_{\Omega}\left[\frac{|u|}{a}+\left|\nabla_{u}\right|\right] \prod_{j=1}^{k-1} \sin ^{j-1} \phi_{j} \prod_{j=k}^{n} \sin ^{a^{+} j-1} \phi_{j} \rho^{a+n} d \rho d \phi .
$$

For $k>m \geq 2$ (3) has the form

$$
\int_{\mathbf{Q}}|u| \prod_{i=1}^{m-1} \sin ^{j-1} \phi_{j} \prod_{j=m}^{k-1} \sin ^{j-2} \phi_{j} \prod_{j=\dot{k}}^{n} \sin ^{a_{+j-2}} \phi_{j} \rho^{a+n-1} d \rho d \phi
$$

$$
\leq K_{m, k} \int_{\Omega}\left[\frac{|u|}{a}+|\nabla u|\right] \prod_{j=1}^{k-1} \sin ^{j-1} \phi_{j} \prod_{j=k}^{n} \sin ^{a_{+j-1}} \phi_{j} \rho^{a_{+} n} d \rho d \phi .
$$


By virtue of the restrictions placed on $\alpha, m$, and $k$ in the statement of the lemma, (4) and (5) are both special cases of

$$
\begin{aligned}
\int_{\mathbf{Q}}|u| & \prod_{j=1}^{i-1} \sin ^{\mu} \phi_{j} \prod_{j=i}^{n} \sin ^{\mu_{j}-1} \phi_{j} \rho^{\alpha_{+n-1}} d \rho d \phi \\
& \leq K \int_{\Omega}\left[\frac{|u|}{a}+|\nabla u|\right] \prod_{j=1}^{n} \sin ^{\mu_{j}} \phi_{j} \rho^{\alpha+n} d \rho d \phi
\end{aligned}
$$

where $1 \leq i \leq n+1$ and $\mu_{j} \geq 0, \mu_{j}>0$ if $j \geq i$. We prove (6) by backwards induction on $i$. For $i=n+1,(6)$ is obtained by applying Lemma 1.2.1 to $u$ considered as a function of $\rho$ and then integrating the remaining variables with the appropriate weights. Assume therefore that (6) has been proved for $i=l+1$ where $1 \leq l \leq n$. If $\beta_{l}<\pi$ we have

$$
\sin \phi_{l} \leq \phi_{l} \leq K_{1} \sin \phi_{l}, \quad \text { if } 0 \leq \phi_{l} \leq \beta_{l} .
$$

Then by Lemma 1.2.1, and since $\left|\partial u / \partial \phi_{l}\right| \leq \rho \Pi_{j=l+1}^{n} \sin \phi_{j}\left|\nabla_{u}\right|$, we have

$$
\begin{aligned}
\int_{0}^{\beta_{l}}|u| \sin ^{\mu} l^{-1} \phi_{l} d \phi_{l} & \leq \int_{0}^{\beta_{l}}|u| \phi_{l}^{\mu^{-1}} d \phi_{l} \\
& \leq K_{2} \int_{0}^{\beta l}\left[|u|+\left|\nabla_{u}\right| \rho \prod_{j=l+1}^{n} \sin \phi_{j}\right] \phi_{l}^{\mu} d \phi_{l} \\
& \leq K_{3} \int_{0}^{\beta} l\left[|u|+\left|\nabla_{u}\right| \rho \prod_{j=l+1}^{n} \sin \phi_{j}\right] \sin ^{\mu} \phi_{l} d \phi_{l} .
\end{aligned}
$$

If $\beta_{l}=\pi$ we obtain (8) by writing $\int_{0}^{\pi}=\int_{0}^{\pi / 2}+\int_{\pi / 2}^{\pi}$ and using, in place of (7), the inequalities

$$
\begin{array}{ll}
\sin \phi_{l} \leq \phi_{l} \leq K_{1}^{\prime} \sin \phi_{l}, & \text { if } 0 \leq \phi_{l} \leq \pi / 2, \\
\sin \phi_{l} \leq \pi-\phi_{l} \leq K_{1}^{\prime \prime} \sin \phi_{l}, & \text { if } \pi / 2 \leq \phi_{l} \leq \pi .
\end{array}
$$

We now have, using (8) and the induction hypothesis,

$$
\begin{aligned}
& \int_{\Omega}|u| \prod_{j=1}^{l-1} \sin ^{\mu} \phi_{j} \prod_{j=l}^{n} \sin ^{\mu_{j}-1} \phi_{j} \rho^{\alpha_{+n-1}} d \rho d \phi \\
& \leq \int_{0}^{a} \rho^{a+n-1} d \rho \prod_{l=1}^{l-1} \int_{0}^{\beta_{j}} \sin ^{\mu}{ }_{j} \phi_{j} d \phi_{j} \prod_{j=l+1}^{n} \int_{0}^{\beta_{j}} \sin ^{\mu{ }_{j}^{-1}} \phi_{j} d \phi_{j} \int_{0}^{\beta_{l}}|u| \sin ^{\mu_{l}-1} \phi_{l} d \phi_{l} \\
& \leq K_{3} \int_{\mathbf{Q}}\left|\nabla_{u}\right| \prod_{j=1}^{n} \sin ^{\mu} \phi_{j} \rho^{a+n} d \rho d \phi \\
& +K_{3} \int_{\mathbf{Q}}|u| \prod_{j=1}^{l} \sin ^{\mu}{ }_{j} \phi_{j} \prod_{j=l+1}^{n} \sin ^{\mu_{j}-1} \phi_{j} \rho^{a_{+n-1}} d \rho d \phi \\
& \leq K \int_{\Omega}\left[\frac{|u|}{a}+\left|\nabla_{u}\right|\right] \prod_{j=1}^{n} \sin ^{\mu} \phi_{j} \rho^{a+n} d \rho d \phi \text {. }
\end{aligned}
$$


This completes the induction establishing (6) and hence the lemma.

We now state without proof a special case, suitable for our purposes, of a well-known combinatorial lemma which is central to one of the standard proofs of the Sobolev Imbedding Theorem. The proof of this lemma may be found in Gagliardo [4, p. 117], or Clark [3].

1.3.2 Lemma. Let $\Omega$ be a domain in $E_{n+1}$ and let $\Omega_{j}, j=1,2, \ldots, n+1$, be the projection of $\Omega$ onto the $n$-dimensional coordinate byperplane orthogonal to the jth coordinate axis in $E_{n+1}$. Let $F_{j}\left(\xi_{1}, \ldots, \xi_{n+1}\right)$ be independent of the coordinate $\xi_{j}$ and suppose $F_{j} \in L^{n}\left(\Omega_{j}\right)$. Then

$$
\left\{\int_{\mathbf{\Omega}} \prod_{j=1}^{n+1}\left|F_{j}(\xi)\right| d \xi\right\}^{n} \leq \prod_{j=1}^{n+1} \int_{\mathbf{\Omega}_{j}}\left|F_{j}(\xi)\right|^{n} d \hat{\xi}_{j}
$$

where $d \hat{\xi}_{j}=d \xi_{1} \cdots d \xi_{j-1} d \xi_{j+1} \cdots d \xi_{n}$.

1.3.3 Theorem. If $p \geq 1,1 \leq k \leq n+1$, and $a>\max (1-k, p-n-1)$ then

(9) $\left\{\int_{\Omega}|u(x)|^{\gamma}\left[r_{k}(x)\right]^{a} d x\right\}^{1 / \gamma} \leq Q\left\{\int_{\Omega}\left[\frac{|u(x)|^{p}}{a^{p}}+|\nabla u(x)|^{p}\right]\left[r_{k}(x)\right]^{a} d x\right\}^{1 / p}$

where $\gamma=(\alpha+n+1) p /(\alpha+n+1-p)$.

Proof. Let $\delta=(\alpha+n) p /(\alpha+n+1-p), q=(\alpha+n) / \alpha, q^{\prime}=(\alpha+n) / n$. We have, by Hölder's inequality and Lemma 1.3.1 (case $m=k$ ),

$$
\begin{aligned}
& \int_{\Omega}|u(x)|^{\gamma}\left[r_{k}(x)\right]^{\alpha} d x \\
& \leq\left\{\int_{\Omega}|u|^{\delta} r_{k}^{a-1} d x\right\}^{1 / q}\left\{\int_{\mathbf{\Omega}}|u|^{(n+1) \delta / n} r_{k}^{(n+1) a / n} d x\right\}^{1 / q^{\prime}} \\
& \quad \leq Q_{1}\left\{\int_{\mathbf{\Omega}}|u|^{\delta-1}\left[\frac{|u|}{a}+|\nabla u|\right] r_{k}^{a} d x\right\}^{1 / q}\left\{\int_{\Omega}|u|^{(n+1) \delta / n} r_{k}^{(n+1) a / n} d x\right\}^{1 / q^{\prime}} .
\end{aligned}
$$

In order to estimate the second integral above we adopt the following notation:

$$
\begin{aligned}
& \rho^{*}=\left(\phi_{1}, \phi_{2}, \ldots, \phi_{n}\right), \\
& \phi_{j}^{*}=\left(\rho, \phi_{1}, \cdots, \hat{\phi}_{j}, \ldots, \phi_{n}\right), \quad j=1,2, \cdots, n
\end{aligned}
$$

where the symbol "a", denotes omission of a component. Set

$$
\begin{aligned}
& \Omega_{0}=\left\{\rho^{*}:\left(\rho, \rho^{*}\right) \in \Omega \text { for } 0<\rho<a\right\}, \\
& \Omega_{j}=\left\{\phi_{j}^{*}:(\rho, \phi) \in \Omega \text { for } 0 \leq \phi_{j}<\beta_{j}\right\},
\end{aligned}
$$


$\Omega_{0}$ and $\Omega_{j}$ are domains in $E_{n}$. We define functions $F_{0}=F_{0}\left(\rho^{*}\right)$ and $F_{j}=$ $F_{j}\left(\phi_{j}^{*}\right)$ as follows:

$$
\begin{aligned}
& F_{0}\left(\rho^{*}\right)=F_{0}\left(\phi_{1}, \cdots, \phi_{n}\right) \\
&=\left\{\sup _{0<\rho<a}\left[|u|^{\delta} \rho^{a+n}\right] \prod_{i=k}^{n} \sin ^{a} \phi_{i} \prod_{i=2}^{n} \sin ^{i-1} \phi_{i}\right\}^{1 / n}, \\
& F_{j}\left(\phi_{j}^{*}\right)=F_{j}\left(\rho, \phi_{1}, \ldots, \hat{\phi}_{j}, \cdots, \phi_{n}\right) \\
&=\left\{\sup _{0 \leq \phi_{j}<\beta_{j}}\left[|u|^{\delta} \sin ^{a+j-1} \phi_{j}\right] \rho^{a+n-1}\right. \\
&\left.\prod_{i=k}^{n} \sin ^{a} \phi_{i} \prod_{i=2}^{j-1} \sin ^{i-1} \phi_{i} \prod_{i=j+1}^{n} \sin ^{i-2} \phi_{i}\right\}^{1 / n}
\end{aligned}
$$

Then we have

$$
|u|^{(n+1) \delta / n_{r}(n+1) a / n} \rho^{n} \prod_{i=2}^{n} \sin ^{i-1} \phi_{i} \leq F_{0}\left(\rho^{*}\right) \prod_{j=1}^{n} F_{j}\left(\phi_{j}^{*}\right) .
$$

Applying Lemma 1.3.2 we obtain

$$
\begin{aligned}
& \int_{\mathbf{\Omega}}|u|^{(n+1)} \delta / n \\
& r_{k}^{(n+1) a / n} d x \\
& \leq \int_{\boldsymbol{\Omega}} F_{0}\left(\rho^{*}\right) \prod_{j=1}^{n} F_{j}\left(\phi_{j}^{*}\right) d \rho d \phi \\
& \leq\left\{\int_{\mathbf{\Omega}_{0}}\left[F_{0}\left(\rho^{*}\right)\right]^{n} d \phi \prod_{j=1}^{n} \int_{\mathbf{\Omega}_{j}}\left[F_{j}\left(\phi_{j}^{*}\right)\right]^{n} d \rho d \hat{\phi}_{j}\right\}^{1 / n} .
\end{aligned}
$$

Now by Lemma 1.2 .3 , and since $|\partial u / \partial \rho| \leq|\nabla u|$

$$
\sup _{0<\rho<a}|u|^{\delta} \rho^{a+n} \leq Q_{2} \int_{0}^{a}|u|^{\delta-1}\left[\frac{|u|}{a}+|\nabla u|\right] \rho^{a+n} d \rho
$$

whence it follows that

$$
\int_{\Omega_{0}}\left[F_{0}\left(\rho^{*}\right)\right]^{n} d \phi \leq Q_{2} \int_{\Omega}|u|^{\delta}-1\left[\frac{|u|}{a}+\left|\nabla_{u}\right|\right] r_{k}^{a} d x
$$

Similarly, by making use of inequalities $(7)$ or $\left(7^{\prime}\right)$ as in Lemma 1.3.1 we obtain from Lemma 1.2.3

$$
\begin{array}{r}
\sup _{0 \leq \phi_{j}<\beta_{j}}|u|^{\delta} \sin ^{\alpha+j-1} \phi_{j} \leq Q_{3, j} \int_{0}^{\beta}|u|^{\delta-1}\left[|u|+\left|\frac{\partial u}{\partial \phi_{j}}\right|\right] \sin ^{\alpha+j-1} \phi_{j} d \phi_{j} \\
\leq Q_{3, j} \int_{0}^{\beta}|u|^{\delta-1}\left[|u|+|\nabla u| \rho \prod_{i=j+1}^{n} \sin \phi_{i}\right] \sin ^{\alpha+j-1} \phi_{j} d \phi_{j}
\end{array}
$$


since $\left|\partial u / \partial \phi_{j}\right| \leq \rho I_{i=j+1}^{n}$ sin $\phi_{i}$. Hence

$$
\begin{aligned}
\int_{\boldsymbol{\Omega}_{j}} & {\left[F_{j}\left(\phi_{j}^{*}\right)\right]^{n} d \rho d \hat{\phi}_{j} } \\
& \leq Q_{3, j} \int_{\Omega}|\nabla u||u|^{\delta-1} r_{k}^{\alpha} d x+Q_{3, j} \int_{\Omega}|u|^{\delta} \frac{r_{k}^{\alpha}}{r_{j+1}} d x \\
& \leq Q_{4, j} \int_{\mathbf{\Omega}}|u|^{\delta-1}\left[\left[\frac{|u|}{a}+\left|\nabla_{u}\right|\right] r_{k}^{a} d x,\right.
\end{aligned}
$$

where we have used Lemma 1.3.1 to obtain the last inequality. Substitution of (12) and (13) into (11) and thence into (10) leads to

$$
\begin{aligned}
& \int_{\mathbf{\Omega}}|u|^{\gamma} r_{k}^{a} d x \leq Q_{5}\left\{\int_{\mathbf{\Omega}}|u|^{\delta-1}\left[\frac{|u|}{a}+|\nabla u|\right] r_{k}^{a} d x\right\}^{1 / q+(n+1) / n q^{\prime}} \\
& \leq Q_{5}\left\{\left\{\int_{\mathbf{\Omega}}|u|^{\gamma} r_{k}^{a} d x\right\}^{(p-1) / p} \cdot\left\{2^{p-1} \int_{\mathbf{\Omega}}\left[\frac{|u|^{p}}{a^{p}}+|\nabla u|^{p}\right] r_{k}^{a} d x\right\}^{1 / p}\right\}^{(a+n+1) /(a+n)} .
\end{aligned}
$$

Since $(\alpha+n) /(\alpha+n+1)-(p-1) / p=1 / \gamma$ the inequality (9) follows immediately.

Remarks. 1. If $a=0$ Theorem 1.3.3 is part of the Sobolev Imbedding Theorem and as such is known to hold for domains $\Omega \subset E_{n+1}$ having the cone property. (See $\$ 2.1$.

2. If $1-k<a \leq p-n-1$ then (9) holds for any $\gamma$ satisfying $1 \leq \gamma<\infty$. It is sufficient to prove this for large $\gamma$. If $\gamma \geq(\alpha+n+1) /(\alpha+n)$ then $\gamma=$ $(a+n+1) q /(\alpha+n+1-q)$ for some $q$ satisfying $1 \leq q<p$. Thus

$$
\begin{aligned}
\left\{\int_{\mathbf{Q}}|u|^{\gamma} r_{k}^{a} d x\right\}^{q / \gamma} & \leq Q \int_{\mathbf{Q}}\left[\frac{|u|^{q}}{a^{q}}+\left|\nabla_{u}\right|^{q}\right] r_{k}^{a} d x \\
& \leq Q\left\{\int_{\mathbf{\Omega}} 2^{(p-q) / q}\left[\frac{|u|^{p}}{a^{p}}+|\nabla u|^{p}\right] r_{k}^{a} d x\right\}^{q / p}\left\{\int_{\mathbf{Q}} r_{k}^{a} d x\right\}^{(p-q) / p}
\end{aligned}
$$

which yields (9) since the last factor on the right is bounded.

3. If $a=m$, a positive integer, then Theorem 1.3 .3 can be proved very simply as follows. Let $y=(x, z)=\left(x_{1}, \ldots, x_{n+1}, z_{1}, \ldots, z_{m}\right)$ denote a point in $E_{n+1+m}$ and define $u^{*}(y)=u(x)$ for $x$ in the domain $\Omega$. If $\Omega^{*}=\left\{y \in E_{n+1+m}\right.$ : $\left.y=(x, z), x \in \Omega, 0<z_{j}<r_{k}(x), 1 \leq j \leq m\right\}$ then $\Omega^{*}$ has the cone property in $E_{n+1+m}$, whence, by Sobolev's theorem, putting $\gamma=(n+1+m) p /(n+1+m-p)$ 


$$
\begin{aligned}
\left\{\int_{\mathbf{\Omega}}|u|^{\gamma} r_{k}^{m} d x\right\}^{1 / \gamma} & =\left\{\int_{\mathbf{\Omega}^{*}}\left|u^{*}(y)\right|^{\gamma} d y\right\}^{1 / \gamma} \leq Q\left\{\int_{\mathbf{\Omega}^{*}}\left[\frac{\left|u^{*}(y)\right|^{p}}{a^{p}}+\left|\nabla u^{*}(y)\right|^{p}\right] d y\right\}^{1 / p} \\
& =Q\left\{\int_{\mathbf{\Omega}}\left[\frac{|u(x)|^{p}}{a^{p}}+|\nabla u(x)|^{p}\right]\left[r_{k}(x)\right]^{m} d x\right\}^{1 / p}
\end{aligned}
$$

since $\left|\nabla_{u}^{*}(y)\right|=|\nabla u(x)|, u^{*}$ being independent of $z$.

4. If $\max (1-k, p-n-1)<a_{1} \leq a \leq a_{2}<\infty$ then the constant $Q$ in (9) can be chosen so as to depend on $\alpha_{1}$ and $\alpha_{2}$ but not on $\alpha$. This can be seen by reviewing the effect of the constants in formulas (1) and (3) of $\$ 1.2$ on the constant $K_{m, k}$ of (3) above, and finally on $Q$. This fact will be useful later.

Theorem 1.3.3 may be generalized in the direction of the corollary to Theorem 1.2 .4 as follows.

1.3.4 Theorem. Let $p \geq 1,1 \leq k \leq n+1$, and $0 \leq s \leq p$. Suppose that $a>$ $\max (1-k+s-s / p, p-n-1)$. Then

(14) $\left\{\int_{\Omega}|u(x)|^{\gamma} s\left[r_{k}(x)\right]^{\alpha-s} d x\right\}^{1 / \gamma} s \leq Q^{*}\left\{\int_{\Omega}\left[\frac{|u(x)|^{p}}{a^{p}}+|\nabla u(x)|^{p}\right]\left[r_{k}(x)\right]^{a} d x\right\}^{1 / p}$ where $\gamma_{s}=(\alpha+n+1-s) p /(\alpha+n+1-p)$.

The proof follows the same lines as that of Theorem 1.3 .3 except that we take

$$
\delta=\frac{(a+n-s) p+s}{a+n+1-p}, \quad q=\frac{(a+n-s) p+s}{(a-s) p+(n+1) s}, \quad q^{\prime}=\frac{(a+n-s) p+s}{n(p-s)}
$$

and replace $\alpha$ in the right-hand side of formula (10) by $\beta=\alpha-s+s / p$. The details are left to the reader.

Of special interest is the case $s=p$, namely

$$
\int_{\mathbf{\Omega}}|u(x)|^{p}\left[r_{k}(x)\right]^{a-p} d x \leq Q^{*} \int_{\mathbf{\Omega}}\left[\frac{|u(x)|^{p}}{a^{p}}+|\nabla u(x)|^{p}\right]\left[r_{k}(x)\right]^{a} d x
$$

which holds for $a>p-k$ and generalizes Theorem 1.2.2. If $r_{k}^{a / p} \in L^{p}\left(\Omega_{\infty}\right)$ where $\Omega_{\infty}=\{(\rho, \phi): 0<\rho<\infty,(a / 2, \phi) \in \Omega\}$ then we obtain, letting $a \rightarrow \infty$ in formula (15),

$$
\int_{\mathbf{\Omega}_{\infty}}|u(x)|^{p}\left[r_{k}(x)\right]^{a-p} d x \leq Q^{*} \int_{\mathbf{\Omega}_{\infty}}|\nabla u(x)|^{p}\left[r_{k}(x)\right]^{a} d x
$$


for $a>p-k$, a generalization of Hardy's inequality.

1.2.5 Example. Let $p \geq 1,1 \leq k \leq n+1$ and suppose that $\alpha>$ $\max (1-k+s-(s / p), p-n-1)$. Let $u(x)=\rho^{-\beta}$ and suppose $\gamma^{\prime}>\gamma_{s}$. It is readily checked that

$$
\int_{\Omega}\left[\frac{|u|^{p}}{a^{p}}+|\nabla u|^{p}\right] r_{k}^{a} d x<\infty
$$

only if $\beta<(\alpha+n+1-p) / p$, and also that

$$
\int_{\mathbf{\Omega}}|u|^{\gamma^{\prime}} r_{k}^{a-s} d x=\infty
$$

if $\beta \geq(\alpha+n+1-s) / \gamma^{\prime}$. Since $(\alpha+n+1-s) / \gamma^{\prime}<(\alpha+n+1-p) / p$. it is possible to choose $\beta$ so that (17) and (18) both hold. This example shows that the exponent $\gamma_{s}$ in (14) (or $\gamma$ in (9)) is the best possible.

1.4 The multi-dimensional case-boundedness and Hölder continuity. We now turn to the case $a \geq 0, a+n+1-p<0$. It is convenient to deal directly with domains $\Omega \subset E_{n+1}$ more general than those considered in $\$ 1.3 . \Omega$ is said to have the "cone property" if there exists a finite cone $C$ (the intersection of an open ball in $E_{n+1}$ centred at the origin, with a set of the form $\{\lambda x: \lambda>0$, $\left.x \in E_{n+1},|x-y|<r\right\}$ where $r>0$ and $y$ is a fixed point in $E_{n+1}$ with $\left.|y|>r\right)$ such that each point $x$ on the boundary $\partial \Omega$ of $\Omega$ is the vertex of a finite cone $C_{x}$ congruent to $C$ and contained in $\Omega$. Also, $\Omega$ has the "strong local Lipschitz property" if each point $x$ on the boundary $\partial \Omega$ has a neighbourhood $U$ such that in some Cartesian coordinate system $\xi$ with origin at $x, \Omega \cap U$ is represented in $U$ by the inequality $\xi_{n+1}<F\left(\xi_{1}, \ldots, \xi_{n}\right)$ with $F$ a Lipschitz continuous function. If $\Omega$ is bounded and has the strong local Lipschitz property then it also has the cone property.

The main results of this section are Theorems 1.4 .2 and 1.4 .3 below. Both are well known if $\alpha=0$, the former being due to Sobolev and the latter to C. B. Morrey. The following lemma will be required in the proof.

1.4.1 Lemma. If $z \in E_{k}$ and $\Omega$ is a domain of finite volume in $E_{k}$, and if $0 \leq a<k$ then

$$
\int_{\Omega}|x-z|^{-a} d x \leq K^{*}(\operatorname{vol} \Omega)^{1-(a / k)}
$$

where the constant $K^{*}=K^{*}(\alpha, k)$ is independent of $z$ and $\Omega$.

The proof of this lemma involves showing that the integral on the left side of (1) does not exceed $\int_{B}|x-z|^{-\alpha} d x$ where $B$ is the ball with centre $z$ and the same volume as $\Omega$. (1) is clearly true for $\Omega=B$. The details may be found in Hellwig [7, p. 53]. 
1.4.2 Theorem. Let $\Omega$ be a domain with the cone property in $E_{n+1}$. Let $1 \leq$ $k \leq n+1$ and let $P$ be an $(n-k+1)$-dimensional byperplane in $E_{n+1}$. Denote by $r(x)$ the distance from $x$ to $P$. If $\alpha \geq 0$ and $\alpha+n+1-p<0$ then for all $u \in C^{1}(\Omega)$ we bave

$$
\sup _{x \in \mathbf{\Omega}}|u(x)| \leq K\left\{\int_{\mathbf{\Omega}}\left[|u(x)|^{p}+|\nabla u(x)|^{p}\right][r(x)]^{\alpha} d x\right\}^{1 / p}
$$

where the constant $K$ may depend on $\alpha, n, p, k$ and the dimensions of the cone $C$ determining the cone property for $\Omega$, but not on $u$.

Proof. Throughout this proof $A_{i}$ and $K_{i}$ will denote various constants depending on one or more of the parameters on which $K$ is allowed to depend in (2). It is sufficient to prove that if $C$ is a finite cone contained in $\Omega$ and having vertex at, say, the origin, then

$$
|u(0)| \leq K\left\{\int_{C}\left[|u(x)|^{p}+|\nabla u(x)|^{p}\right][r(x)]^{\alpha} d x\right\}^{1 / p} .
$$

For $0 \leq j \leq n+1$ let $A_{j}$ denote the supremum of the $j$-dimensional Lebesgue measure of the projection of $C$ onto $E_{j}$, taken over all $j$-dimensional subspaces $E_{j}$ of $E_{n+1}$. Writing $x=\left(x^{\prime}, x^{\prime \prime}\right)$ where $x^{\prime}=\left(x_{1}, \ldots, x_{n+1-k}\right)$ and $x^{\prime \prime}=\left(x_{n+2-k}, \ldots\right.$, $x_{n+1}$ ) we may assume, without loss of generality, that $P$ is orthogonal to the coordinate axes corresponding to the components of $x^{\prime \prime}$. Define

$$
\begin{aligned}
Q & =\left\{x^{\prime} \in E_{n+1-k}:\left(x^{\prime}, x^{\prime \prime}\right) \in C \text { for some } x^{\prime \prime} \in E_{k}\right\}, \\
R\left(x^{\prime}\right) & =\left\{x^{\prime \prime} \in E_{k}:\left(x^{\prime}, x^{\prime \prime}\right) \in C\right\}, \text { for each } x^{\prime} \in Q .
\end{aligned}
$$

For $0 \leq t \leq 1$ we denote by $C_{t}$ the cone $\{t x: x \in C\}$ so that $C_{t} \subseteq C$ with equality if $t=1$. For $C_{t}$ we define the quantities $A_{t, j}, Q_{t}$ and $R_{t}\left(x^{\prime}\right)$ analogously to the similar quantities defined above for $C$. Clearly $A_{t, j}=t^{j} A_{j}$. If $x \in C$ we have $u(x)=u(0)+\int_{0}^{1}(d / d t) u(t x) d t$ so that $|u(0)| \leq|u(x)|+|x| \int_{0}^{1}\left|\nabla_{u}(t x)\right| d t$. Setting $V=\operatorname{vol} C$ and $a=\sup _{x \in C}|x|$, and integrating the above inequality over $C$, we obtain

$$
\begin{aligned}
V|u(0)| & \leq \int_{C}|u(x)| d x+a \int_{C} d x \int_{0}^{1}\left|\nabla_{u}(t x)\right| d t \\
& =\int_{C}|u(x)| d x+a \int_{0}^{1} t^{-n-1} d t \int_{C_{t}}\left|\nabla_{u}(x)\right| d x .
\end{aligned}
$$

Let $z$ denote the projection of $x$ onto $P$. Then $r(x)=\left|x^{\prime \prime}-z^{\prime \prime}\right|$. Since $\alpha \geq 0$ and $a+n+1-p<0$ we have $p>1$ and so by Lemma 1.4.1

$$
\begin{aligned}
& \int_{C_{t}}[r(x)]^{-a /(p-1)} d x=\int_{Q_{t}} d x^{\prime} \int_{R_{t}\left(x^{\prime}\right)}\left|x^{\prime \prime}-z^{\prime \prime}\right|^{-a /(p-1)} d x^{\prime \prime} \\
& \leq K_{1} \int_{Q_{t}}\left[A_{t, k}\right]^{1-\alpha / k(p-1)} d x^{\prime}=K_{1}\left[A_{t, k}\right]^{1-\alpha / k(p-1)} A_{t, n+1-k} \\
& =K_{2} t^{n+1-\alpha /(p-1)} \text {. }
\end{aligned}
$$


It follows that

$$
\begin{aligned}
\int_{C_{t}}\left|\nabla_{u}(x)\right| d x & \leq\left\{\int_{C_{t}}\left|\nabla_{u}(x)\right|^{p}[r(x)]^{a} d x\right\}^{1 / p}\left\{\int_{C_{t}}[r(x)]^{-a /(p-1)} d x\right\}^{(p-1) / p} \\
& \leq K_{3} t^{n+1-(a+n+1) / p}\left\{\int_{C}\left|\nabla_{u}(x)\right|^{p}[r(x)]^{a} d x\right\}^{1 / p}
\end{aligned}
$$

and so, since $a+n+1<p$

$$
\int_{0}^{1} t^{-n-1} d t \int_{C_{t}}|\nabla u(x)| d x \leq K_{4}\left\{\int_{C}\left|\nabla_{u}(x)\right|^{p}[r(x)]^{a} d x\right\}^{1 / p} .
$$

Similarly

$$
\begin{aligned}
\int_{C}|u(x)| d x & \leq\left\{\int_{C}|u(x)|^{p}[r(x)]^{\alpha} d x\right\}^{1 / p}\left\{\int_{C}[r(x)]^{-\alpha /(p-1)} d x\right\}^{(p-1) / p} \\
& \leq K_{5}\left\{\int_{C}|u(x)|^{p}[r(x)]^{\alpha} d x\right\}^{1 / p}
\end{aligned}
$$

Inequality (3) now follows from (4), (6) and (7) and the proof is complete.

1.4.3 Theorem. Suppose that all the conditions of Theorem 1.4 .2 are satis. fied, and that in addition $\Omega$ is bounded and has the strong local Lipschitz prop. erty. Then for all $u \in C^{1}(\Omega)$ we have

$$
\sup _{x, y \in \mathbf{\Omega}} \frac{|u(x)-u(y)|}{|x-y|^{\mu}} \leq K^{\prime}\left\{\int_{\Omega}\left[|u(x)|^{p}+\left|\nabla_{u}(x)\right|^{p}\right][r(x)]^{a} d x\right\}^{1 / p}
$$

where $\mu=1-(a+n+1) / p$ satisfies $0<\mu<1$ and $K^{\prime}$ is independent of $u$.

Proof. By virtue of the previous theorem it is sufficient to prove (8) for sufficiently small $|x-y|$.

First assume that $\Omega$ is a cube, which we may also assume without loss of generality to have unit side. For $0<t<1$ let $\Omega_{t}$ be a cube of side $t$ with faces parallel to those of $\Omega$ and such that $\bar{\Omega}_{t} \subset \Omega$. In the same way we obtained (5) above we can show that

$$
\int_{\mathbf{\Omega}_{t}}\left|\nabla_{u}(z)\right| d z \leq K_{6} t^{n+1-(a+n+1) / p}\left\{\int_{\mathbf{Q}}|\nabla u(z)|^{p}[r(z)]^{a} d z\right\}^{1 / p} .
$$

Let $x, y \in \Omega,|x-y|=\rho<1$. Then there exists a fixed cube $\Omega_{\rho}$ with $\bar{\Omega}_{\rho} \subset \Omega$ such that $x, y \in \bar{\Omega}_{\rho}$. If $z \in \Omega_{\rho}, u(x)=u(z)-\int_{0}^{1}(d / d t) u(x+t(z-x)) d t$ so that

$$
|u(x)-u(z)| \leq \rho \int_{0}^{1}|\nabla u(x+t(z-x))| d t .
$$

Hence 


$$
\begin{aligned}
\mid u(x)-\frac{1}{\rho^{n+1}} & \int_{\Omega_{\rho}} u(z) d z \mid \\
& \leq \frac{1}{\rho^{n}} \int_{\Omega_{\rho}} d z \int_{0}^{1}|\nabla u(x+t(z-x))| d t \\
& =\frac{1}{\rho^{n}} \int_{0}^{1} t^{-n-1} d t \int_{\Omega_{t \rho}}|\nabla u(z)| d z \\
& \leq K_{6} \rho^{\mu} \int_{0}^{1} t^{-(a+n+1) / p} d t\left\{\int_{\Omega}|\nabla u(z)|^{p}[r(z)]^{a} d z\right\}^{1 / p} \\
& \leq K_{7} \rho^{\mu}\left\{\int_{\Omega}\left|\nabla_{u}(z)\right|^{p}[r(z)]^{a} d z\right\}^{1 / p}
\end{aligned}
$$

since $a+n+1<p$. A similar inequality holds with $x$ replaced by $y$ and so

$$
|u(x)-u(y)| \leq 2 K_{7}|x-y|^{\mu}\left\{\int_{\Omega}|\nabla u(z)|^{p}[r(z)]^{\alpha} d z\right\}^{1 / p} .
$$

We have thus proved ( 8 ) for a cube, and hence, via a nonsingular linear transformation, for a parallelepiped.

Now suppose $\Omega$ is bounded and has the strong local Lipschitz property. Then there exists a finite open cover $\left\{U_{i}\right\}$ of $\bar{\Omega}$ and corresponding parallelepipeds $\left\{\pi_{i}\right\}$ each having one vertex at 0 such that $x+\pi_{i} \subset \Omega$ for each $x \in U_{i} \cap \bar{\Omega}$. Also, there exist constants $\delta_{0}$ and $\delta_{1}$ such that for any $x, y \in \Omega$ with $|x-y|<\delta_{0}$ there exists $U_{i}$ such that $x, y \in U_{i}$, and moreover there exists $z \in\left(x+\pi_{i}\right) \cap$ $\left(y+\pi_{i}\right)$ with $|x-z|+|y-z| \leq \delta_{1}|x-y|$. This last assertion is most easily seen by supposing $\pi_{i}$ is a cube and then applying a nonsingular linear transformation. The required inequality (8) now follows for $|x-y|<\delta_{0}$ from the case where $\Omega$ is a parallelepiped.

Remarks. 1. If $\Omega$ is bounded both of the above theorems hold also for the case $a<0, n+1<p$. For instance, from Theorem 1.4 .2 with $\alpha=0$ we obtain

$$
\sup _{x \in \boldsymbol{\Omega}}|u(x)| \leq K\left\{\int_{\Omega}\left[|u(x)|^{p}+|\nabla u(x)|^{p}\right] d x\right\}^{1 / p} .
$$

Since $\alpha<0$ and $r(x)$ is bounded in $\Omega$ we have $[r(x)] \geq$ constant, whence (2) follows from (10). A similar argument establishes (8).

2. If $\alpha$ is a positive integer both theorems follow from their well-known special cases $\alpha=0$ by simple arguments similar to that used in Remark 3 following Theorem 1.3.3. 


\section{PART II. IMBEDDING AND NONIMBEDDING THEOREMS FOR SOBOLEV SPACES}

2.1. The Sobolev Imbedding Theorem. Let $\Omega$ be an open domain in $E_{n}$. The Sobolev space $W^{m, p}(\Omega)$ is, for $m=1,2, \ldots$ and $p \geq 1$, the space of all (possibly complex -valued) functions $u$ in $L^{p}(\Omega)$ whose distributional partial derivatives of orders up to and including $m$ also belong to $L^{p}(\Omega) . W^{m, p}(\Omega)$ is a Banach space with respect to the norm

$$
\left|u: W^{m, p}(\Omega)\right|=\left\{\sum_{0 \leq|s| \leq m}\left|D^{s} u: L^{p}(\Omega)\right|^{p}\right\}^{1 / p}
$$

where $\left|u: L^{p}(\Omega)\right|=\left\{\int_{\Omega}|u(x)|^{p} d x\right\}^{1 / p}$ is the norm in $L^{p}(\Omega)$. Here $s=\left(s_{1}, s_{2}, \ldots, s_{n}\right)$ is an $n$-tuple of nonnegative integers; $|s|=s_{1}+\cdots+s_{n} ; D^{s}=\left(\partial / \partial x_{1}\right)^{s_{1}} \ldots$ $\left(\partial / \partial x_{n}\right)^{s} n$; and $d x=d x_{1} \cdots d x_{n}$ is the Lebesgue volume element in $E_{n}$. It is well known (see [10]) that the space of all functions $u$ in $C^{\infty}(\Omega)$ for which the norm is finite is dense in $W^{m, p}(\Omega)$.

The Sobolev Imbedding Theorem (Theorem 2.1.1 below) establishes the imbeddings (continuous injections) of $W^{m, p}(\Omega)$ into various Lebesgue and continuous function spaces over $\Omega$. Required for the proof is some form of regularity hypothesis on $\Omega$. The most common, and weakest, of these hypotheses is that $\Omega$ should have the cone property. For certain imbeddings the slightly stronger strong local Lipschitz property is required. (For definitions see the beginning of \$1.4.)

For $j=0,1,2, \ldots$ we denote by $C^{j}(\bar{\Omega})$ the space of functions which, together with all their partial derivatives of orders up to and including $j$, are continuous and bounded on $\Omega \cdot C^{j}(\bar{\Omega})$ is a Banach space with respect to the norm

$$
\left|u: C^{j}(\bar{\Omega})\right|=\max _{0 \leq|s| \leq j} \sup _{x \in \Omega}\left|D^{s} u(x)\right|
$$

In addition, for $0<\nu \leq 1$ we denote by $C^{j, \nu}(\bar{\Omega})$ the subspace of $C^{j}(\bar{\Omega})$ consisting of those functions whose $j$ th order partial derivatives satisfy in $\Omega$ a Hölder condition of exponent $\nu . C^{j, \nu}(\bar{\Omega})$ is a Banach space with respect to the norm

$$
\left|u: C^{j, \nu}(\bar{\Omega})\right|=\left|u: C^{j}(\bar{\Omega})\right|+\max _{|s|=j x, y \in \Omega, x \neq y} \sup _{|x-y|^{\nu}} \frac{\left|D^{s} u(x)-D^{s} u(y)\right|}{\mid x .}
$$

We denote by $A \rightarrow B$ the imbedding (continuous injection) of the normed linear space $A$ into the normed linear space $B$; i.e. $A \rightarrow B$ signifies $A \subset B$ and $|u: B| \leq$ const $|u: A|$ for all $u \in A$.

2.1.1 Theorem (The Sobolev Imbedding Theorem). Let $\Omega$ have the cone property. Then there exist imbeddings of the following types: 
(i) if $m p<n$ and $p \leq q \leq n p /(n-m p)$ then $W^{m, p}(\Omega) \rightarrow L^{q}(\Omega)$;

(ii) if $m p=n$ and $p \leq q<\infty$ then $W^{m, p}(\Omega) \rightarrow L^{q}(\Omega)$, if also $p=1$ then $W^{n, 1}(\Omega) \rightarrow C^{0}(\bar{\Omega})$

(iii) if $(m-j) p>n$ for some $j=0,1,2, \ldots$ then $W^{m, p}(\Omega) \rightarrow C^{j}(\bar{\Omega})$.

If, in addition, $\Omega$ bas the strong local Lipschitz property then case (iii) may be refined as follows:

(iiia) if $(m-j-1) p<n<(m-j) p$ and $0<\nu \leq((m-j) p-n) / p$ then $W^{m, p}(\Omega)$ $\rightarrow C^{j, \nu}(\bar{\Omega})$;

(iiib) if $(m-j-1) p=n$ and $0<\nu<1$ then $W^{m, p}(\Omega) \rightarrow C^{j, \nu}(\bar{\Omega})$.

Remarks. 1. Parts (i), (ii) and (iii) are due essentially to S. L. Sobolev [12] while the refinements (iiia) and (iiib) are due to C. B. Morrey [11]. For a definitive presentation of the proof of this theorem, the reader is referred to Gagliardo [4] or Clark [3]. Not included in our statement of the theorem are certain trace imbeddings of $W^{m, p}(\Omega)$ into $L^{q}$ spaces defined over lower dimensional manifolds contained in $\Omega$. There are numerous generalizations of Theorem 2.1.1 (mostly Russian) to various other spaces, often involving weighted norms or fractional order derivatives. The theorem and its generalizations are useful in the study of partial differential operators on $\Omega$.

2. In asserting the imbedding of $W^{m, p}(\Omega)$ into a continuous function space such as $C^{j}(\bar{\Omega})$ or $C^{j, \nu}(\bar{\Omega})$ it is understood that a function $u \in W^{m, p}(\Omega)$ may require redefinition on a set of measure zero in order to belong to the continuous function space. The elements of $W^{m, p}(\Omega)$ are, strictly speaking, equivalence classes of almost everywhere equal functions and existence of the imbedding indicates that an element $\tilde{u}$ of the class $u \in W^{m, p}(\Omega)$ belongs to the continuous function space in question.

3. It is obvious that if $\Omega$ has finite volume and $1 \leq q \leq p$ then $W^{m, p}(\Omega) \rightarrow$ $L^{q}(\Omega)$. It is shown in [1] that no imbedding of the above form is possible for any $q<p$ unless $\Omega$ has finite volume.

4. Examples can be given to show that the imbeddings of Theorem 2.1.1 are "best possible" in the sense that no other range spaces of the same type as the ones specified can be used in place of the ones specified. E.g. if $m p<n$ and $q>n p /(n-m p)$ then $W^{m, p}(\Omega) \nrightarrow L^{q}(\Omega)$. In case (ii) however, a certain Orlicz space can be shown to be the natural range of the imbedding (see Trudinger [13]).

Many interesting domains fail to have the cone property. Domains whose boundaries consist entirely of $(n-1)$-dimensional surfaces may so fail if the boundary has cusps, or, even if smooth, if the domain is unbounded and narrow at infinity. It is our purpose here to examine the possibility of establishing imbeddings of the type considered in Theorem 2.1.1 for certain classes of domains not having the cone property. 
$\S 2.2$ is concerned with unbounded domains which become narrow at infinity. We show that generally no imbeddings of the desired type are possible.

$\$ 2.3$ is concerned with classes of domains having cusps. We show that if these cusps have "power sharpness" Theorem 2.1.1 survives but with weakened conclusions, establishing imbeddings of all three types for a large, though by no means exhaustive, class of domains with such cusps. Our results sharpen and generalize certain similar results obtained by I. Globenko ([5], [6]) by different methods. Finally we show that no imbeddings of the desired types are possible if the domain has cusps of "exponential sharpness", i.e. cusps sharper than any power cusp.

2.2 Unbounded domains-a nonimbedding theorem. An unbounded domain $\Omega$ in $E_{n}$ may have a smooth boundary and still fail to satisfy the cone condition if it becomes narrow at infinity. For unbounded $\Omega$ let $\Omega_{N}$ denote the set $\{x \in \Omega$ : $N \leq|x| \leq N+1\}$. The writer and John Fournier have shown in [1] that if there is any imbedding of the form

$$
W^{m, p}(\Omega) \rightarrow L^{q}(\Omega)
$$

where $q>p$ then either

(a) vol $\Omega=\infty$ and $\overline{\lim }_{N \rightarrow \infty}$ vol $\Omega_{N}>0$, or

(b) vol $\Omega<\infty$ and $\lim _{N \rightarrow \infty} e^{k N}$ vol $\Omega_{N}=0$ for any $k$.

Unbounded domains with the cone property fall under the alternative (a).

Example. The domain $\Omega=\left\{(x, y) \in E_{2}: x>0,0<y<e^{-x^{2}}\right\}$ satisfies (b) above. However, the function $u(x, y)=e^{x^{2} / q}$ is easily seen to belong to $W^{m, p}(\Omega)$ for $1 \leq p<q$ and any $m$, but not to $L^{q}(\Omega)$.

This example leads us to speculate that there are no unbounded domains in class (b) above for which (1) holds for some $q>p$. Such a result was proved for connected $\Omega$ and $m=1$ by R. Andersson [2]. We prove it in general.

2.2.1 Theorem. If $\Omega$ is unbounded and has finite volume there exist no imbeddings of type (1) for any $q>p$.

Proof. The method of proof is suggested by the example given above. We construct a function $u(x)$ depending only on the distance of $x$ from the origin, whose growth is rapid enough to prevent membership in $L^{q}(\Omega)$ but still slow enough to allow membership in $W^{m, p}(\Omega)$.

Let $A(r)$ denote the surface area (Lebesgue $(n-1)$-measure) of the intersection of $\Omega$ with the spherical surface of radius $r$ centred at the origin. Then

$$
\int_{0}^{\infty} A(r) d r=\operatorname{vol} \Omega<\infty
$$

Without loss of generality we may assume that vol $\Omega=1$. We define numbers $r_{n}$ $(n=0 ; 1,2, \ldots)$ by 


$$
\int_{r_{n}}^{\infty} A(r) d r=2^{-n}=\int_{r_{n-1}}^{r} A(r) d r
$$

so that clearly $r_{0}=0$ and $r_{n} \uparrow \infty$ as $n \rightarrow \infty$. Let $\Delta r_{n}=r_{n+1}-r_{n}$ and fix $\epsilon$ such that $0<\epsilon<(m p)^{-1}-(m q)^{-1}$. There must exist an increasing sequence $\left\{n_{j}\right\}_{j=1}^{\infty}$ such that $\Delta r_{n_{j}} \geq 2^{-\epsilon n_{j}}$ for otherwise $\Delta r_{n}<2^{-\epsilon n}$ for all but possibly finitely many $n$ whence $\sum_{n=0}^{\infty} \Delta r_{n}<\infty$, a contradiction. For convenience we assume $n_{1} \geq 1$ so that $n_{j} \geq j$ for all $j$. We denote $a_{0}=0, a_{j}=r_{n_{j}+1}, b_{j}=r_{n_{j}}(j=1,2, \ldots)$, and note that $a_{j-1} \leq b_{j}<a_{j}$ and $a_{j}-b_{j}=\Delta r_{n_{j}} \geq 2^{-n_{j}}$.

Let $f$ be a fixed, nonnegative, infinitely differentiable function on $(-\infty, \infty)$ with the properties

(i) $0 \leq f(t) \leq 1$ for all $t$,

(ii) $f(t)=0$ if $t \leq 0 ; f(t)=1$ if $t \geq 1$,

(iii) $f^{(k)}(t) \leq M$ for all $t$ if $1 \leq k \leq m$.

For $x$ in $\Omega$ let $r=|x|$ and define a function $u$ in $C^{\infty}(\Omega)$ as follows (taking $\left.n_{0}=0\right)$

$$
\begin{array}{ll}
u(x)=2^{n_{j-1} / q} & \text { for } a_{j-1} \leq r \leq b_{j} \\
u(x)=2^{n_{j-1} / q}+\left(2^{n_{j} / q}-2^{n_{j-1} / q}\right) f\left(\left(r-b_{j}\right) /\left(a_{j}-b_{j}\right)\right) & \text { for } b_{j} \leq r \leq a_{j}
\end{array}
$$

Denoting $\Omega_{j}=\left\{x \in \Omega: a_{j-1} \leq|x| \leq a_{j}\right\}$ we have

$$
\begin{aligned}
& \int_{\mathbf{\Omega}_{j}}|u(x)|^{p} d x=\left\{\int_{a_{j-1}}^{b_{j}}+\int_{b_{j}}^{a_{j}}\right\}[u(x)]^{p} A(r) d r \\
& \leq 2^{n}{ }_{j-1}^{p / q} \int_{a_{j-1}}^{\infty} A(r) d r+2^{n}{ }^{p / q} \int_{b_{j}}^{a_{j}} A(r) d r \\
& =1 / 2\left[2^{-n}{ }_{j-1^{(1-p / q)}}+2^{-n_{j}(1-p / q)}\right]=2^{-(j-1)(1-p / q)} .
\end{aligned}
$$

Since $p<q$ this forces $\int_{\mathbf{\Omega}}|u(x)|^{p} d x=\sum_{j=1}^{\infty} \int_{\Omega_{j}}|u(x)|^{p} d x<\infty$ and so $u \in L^{p}(\Omega)$. To prove that $u \in W^{m, p}(\Omega)$ it is now sufficient to show that $d^{k} u / d r^{k} \in L^{p}(\Omega)$ for $1 \leq k \leq m$. We have

$$
\begin{aligned}
\int_{\Omega_{j}}\left|\frac{d^{k}}{d r^{k}} u(x)\right|^{p} d x=\int_{b_{j}}^{a}\left|\frac{d^{k} u}{d r^{k}}\right|^{p} A(r) d r \\
\leq M^{p} \frac{2^{n}{ }^{p / q}}{\left[a_{j}-b_{j}\right]^{k p}} \int_{b_{j}}^{a_{j}} A(r) d r \\
\quad=1 / 2 M^{p} 2^{-n_{j}\left(1-p / q-\epsilon_{k p)}\right.} \leq 1 / 2 M^{p} 2^{-C_{j}}
\end{aligned}
$$


where $C=1-p / q-\epsilon k p>0$ since $\epsilon<1 / m p-1 / m q$. It follows that $\int_{\mathbf{Q}}\left|d^{k} u / d r^{k}\right|^{p} d x$ $<\infty$. Finally, we note that

$$
\begin{aligned}
& \int_{\Omega_{j}}|u(x)|^{q} d x \geq 2^{n_{j-1}} \int_{a_{j-1}}^{a_{j}} A(r) d r \\
& =2^{n_{j-1}}\left[2^{-n_{j-1}-1}-2^{-n_{j}-1}\right] \geq 1 / 4
\end{aligned}
$$

whence $\int_{\Omega}|u(x)|^{q} d x=\infty$. Since $u$ belongs to $W^{m, p}(\Omega)$ but not to $L^{q}(\Omega)$ the theorem is proved.

Remarks. 1. Following the discussion at the beginning of this section Theorem 2.2.1 has the force of precluding the existence of imbeddings of type (1) for any $q>p$ whenever $\Omega$ is unbounded and satisfies $\lim _{N \rightarrow \infty}$ vol $\Omega_{N}=0$, a condition obviously much weaker than finite volume.

2. Since the counterexample function $u$ constructed in the proof of the above theorem is unbounded it serves also to show that there can be no imbedding of $W^{m, p}(\Omega)$ into $C^{j}(\bar{\Omega})$ for any $j$ (if $\Omega$ is unbounded and has finite volume.)

2.3 Domains with cusps. Let it be assumed from the outset that each domain $\Omega \subset E_{n}$ considered in this section has boundary $\partial \Omega$ consisting of $(n-1)$-dimensional surfaces, and that $\Omega$ lies on only one side of $\partial \Omega$. $\Omega$ is said to have a cusp at $x_{0} \in \partial \Omega$ if no finite cone of positive volume contained in $\Omega$ can have vertex at $x_{0}$. The failure of a domain $\Omega$ to have any cusps does not, of course, guarantee that the domain has the cone property.

We begin by considering cusps of power sharpness.

2.3.1 Definition. For $1 \leq k \leq n-1$ and $\lambda \geq 1$ we denote by $\Omega_{k, \lambda}$ the standard power cusp domain in $E_{n}$ specified by the inequalities:

$$
\begin{aligned}
& x_{1}^{2}+\cdots+x_{k}^{2}<x_{k+1}^{2 \lambda}, \\
& x_{k+1}>0, \cdots, x_{n}>0, \\
& {\left[x_{1}^{2}+\cdots+x_{k}^{2}\right]^{1 / \lambda}+x_{k+1}^{2}+\cdots+x_{n}^{2}<a^{2}}
\end{aligned}
$$

where $a$ is the radius of the ball of unit volume in $E_{n}$. Clearly $a<1 . \Omega_{k, \lambda}$ has axial plane spanned by the $x_{k+1}, \cdots, x_{n}$ coordinate axes, and vertical plane spanned by $x_{k+2}, \cdots, x_{n}$. If $k=n-1$ the origin is the only vertex point of $\Omega_{k, \lambda}$. The outer boundary surface (as determined by (1)) is taken to be of this form in order to simplify calculations later. It could be taken to be a sphere or more general surface bounded away from the origin. 
Example. Let $n=3 . \Omega_{2,2}$ is the domain in $E_{3}$ specified in cylindrical polar coordinates $(r, \theta, z)$ by

$$
r<z^{2}, \quad z>0, \quad r+z^{2}<[3 / 4 \pi]^{2 / 3} \text {. }
$$

It has a simple cusp at the origin. $\Omega_{1,2}$ is the domain in $E_{3}$ specified in Cartesian coordinates by

$$
|x|<y^{2}, \quad y>0, \quad z>0, \quad|x|+y^{2}+z^{2}<[3 / 4 \pi]^{2 / 3} .
$$

This domain has a one-dimensional cusp line along the $z$-axis.

Together with $\Omega_{k, \lambda}$ we shall consider the associated standard cone $\Omega_{k, 1}$ which is a domain of the type considered in $\$ 1.3 . \Omega_{k, 1}$ is specified in Cartesian coordinates $y_{1}, \cdots, y_{n}$ by

$$
\begin{aligned}
& y_{1}^{2}+\cdots+y_{k}^{2}<y_{k+1}^{2}, \\
& y_{k+1}>0, \cdots, y_{n}>0 \\
& y_{1}^{2}+\cdots+y_{n}^{2}<a^{2} .
\end{aligned}
$$

It is convenient to adopt generalized "cylindrical" coordinates $\left(r_{k}, \phi_{1}, \ldots\right.$, $\left.\phi_{k-1}, y_{k+1}, \cdots, y_{n}\right)$ in $E_{n}$ so that $r_{k} \geq 0,-\pi \leq \phi_{1} \leq \pi, 0 \leq \phi_{2}, \cdots, \phi_{k-1} \leq \pi$ and

$$
\begin{aligned}
& y_{1}=r_{k} \sin \phi_{1} \sin \phi_{2} \cdots \sin \phi_{k-1}, \\
& y_{2}=r_{k} \cos \phi_{1} \sin \phi_{2} \cdots \sin \phi_{k-1}, \\
& y_{3}=r_{k} \cos \phi_{2} \cdots \sin \phi_{k-1}, \\
& \cdot \\
& \cdot \\
& y_{k}=r_{k} \cos \phi_{k-1} .
\end{aligned}
$$

In terms of these coordinates $\Omega_{k, 1}$ is represented by

$$
\begin{gathered}
0 \leq r_{k}<y_{k+1}, \quad y_{k+1}>0, \cdots, y_{n}>0, \\
r_{k}^{2}+y_{k+1}^{2}+\cdots+y_{n}^{2}<a^{2} .
\end{gathered}
$$

The standard cusp $\Omega_{k, \lambda}$ may be transformed into the associated cone $\Omega_{k, 1}$ by means of the one-to-one transformation 


$$
\begin{aligned}
& x_{1}=r_{k}^{\lambda} \sin \phi_{1} \sin \phi_{2} \cdots \sin \phi_{k-1}, \\
& x_{2}=r_{k}^{\lambda} \cos \phi_{1} \sin \phi_{2} \cdots \sin \phi_{k-1}, \\
& \cdot \\
& \cdot \\
& x_{k}=r_{k}^{\lambda} \cos \phi_{k-1}, \\
& x_{k+1}=y_{k+1}, \\
& \cdot \\
& \cdot \\
& x_{n}=y_{n}
\end{aligned}
$$

which has Jacobian determinant $\left|\partial\left(x_{1}, \ldots, x_{n}\right) / \partial\left(y_{1}, \cdots, y_{n}\right)\right|=\lambda r_{k}^{(\lambda-1) k}$.

2.3.2 Lemma. Let $\alpha^{*} \geq 0$. If $\alpha^{*}>p-n$ let $1 \leq \gamma \leq\left(\alpha^{*}+n\right) p /\left(\alpha^{*}+n-p\right)$; otberwise let $1 \leq \gamma<\infty$. There exists a constant $\bar{K}=\bar{K}\left(n, p, a^{*}\right)$ such that if $1 \leq k \leq n-1$ and $\lambda \geq 1$ satisfy $a \equiv(\lambda-1) k \leq \alpha^{*}$ then for all $u \in C^{1}\left(\Omega_{k, \lambda}\right)$

$$
\left\{\int_{\mathbb{\Omega}_{k, \lambda}}|u(x)|^{\gamma} d x\right\}^{.1 / \gamma} \leq K\left\{\int_{\Omega_{k, \lambda}}\left[|u(x)|^{p}+|\nabla u(x)|^{p}\right] d x\right\}^{1 / p} .
$$

Proof. We first establish (4) for given $k$ and $\lambda$ and then show that $K$ can be chosen to be independent of these parameters. Suppose $a^{*}>p-n$. It is sufficient to prove (4) for $\gamma=\left(a^{*}+n\right) p /\left(a^{*}+n-p\right)$. For $u \in C^{1}\left(\Omega_{k, \lambda}\right)$ define $\tilde{u}(y)=u(x)$ where $y$ is related to $x$ by (2) and (3). Thus $\tilde{u} \in C^{1}\left(\Omega_{k, 1}\right)$ and so by Theorem 1.3.3 and since $\gamma \leq(\alpha+n) p /(\alpha+n-p)$ we have

$$
\begin{aligned}
\left\{\int_{\mathbf{\Omega}_{k, \lambda}}|u(x)|^{\gamma} d x\right\}^{1 / \gamma}=\left\{\lambda \int_{\mathbf{a}_{k, 1}}|u(y)|^{\gamma}\left[r_{k}(y)\right]^{\alpha} d y\right\}^{1 / \gamma} \\
\leq K_{1}\left\{\int_{\mathbf{a}_{k, 1}}\left[|u(y)|^{p}+\left|\nabla_{u}(y)\right|^{p}\right]\left[r_{k}(y)\right]^{\alpha} d y\right\}^{1 / p} .
\end{aligned}
$$

Now $x_{j}=r_{k}^{\lambda-1} y_{j}$ if $1 \leq j \leq k ; x_{j}=y_{j}$ if $k+1 \leq j \leq n$. Since $r_{k}^{2}=y_{1}^{2}+\cdots+y_{k}^{2}$ we have

$$
\frac{\partial x_{j}}{\partial y_{i}}= \begin{cases}\delta_{i j} r_{k}^{\lambda-1}+(\lambda-1) r_{k}^{\lambda-3} y_{i} y_{j} & \text { if } 1 \leq i, j \leq k \\ \delta_{i j} & \text { otherwise. }\end{cases}
$$


Since $r_{k}(y) \leq 1$ on $\Omega_{k, 1}$ it follows that $|\nabla \widetilde{u}(y)| \leq K_{2}|\nabla u(x)|$. Hence (4) follows from (5) in this case. For $\alpha^{*} \leq p-n$ and any $\gamma$ the proof is similar, and uses the second remark following Theorem 1.3.3.

In order to show that the constant $K$ in (4) can be chosen to be independent of $k$ and $\lambda$ provided $\alpha=(\lambda-1) k \leq \alpha^{*}$ we note that it is sufficient to prove that there is a constant $J$ such that for any $k, \lambda$ with $1 \leq k \leq n-1, \alpha \leq \alpha^{*}$, and all $v \in C^{1}\left(\Omega_{k, 1}\right)$

$$
\left\{\int_{\Omega_{k, 1}}|v(y)|^{\gamma}\left[r_{k}(y)\right]^{a} d y\right\}^{1 / \gamma} \leq J\left\{\int_{\Omega_{k, 1}}\left[|v(y)|^{p}+\left|\nabla_{\nu}(y)\right|^{p}\right]\left[r_{k}\right]^{a} d y\right\}^{1 / p} .
$$

In fact it is sufficient to prove (6) with $J$ depending on $k$ as we can then maximize $J(k)$ over the finitely many allowed values of $k$. We distinguish three cases.

Case 1. $\alpha^{*}<p-n, 1 \leq \gamma<\infty$. By Theorem 1.4 .2 we have for $0 \leq a \leq a^{*}$

$$
\sup _{x \in \mathbf{\Omega}_{k, 1}}|v(x)| \leq J^{*}(\alpha)\left\{\int_{\boldsymbol{\Omega}_{k, 1}}\left[|v(y)|^{p}+|\nabla v(y)|^{p}\right]\left[r_{k}(y)\right]^{\alpha} d y\right\}^{1 / p} .
$$

Since the integral on the right increases as $\alpha$ decreases we have $J^{*}(\alpha) \leq J^{*}\left(\alpha^{*}\right)$ and (6) then follows from (7) and the boundedness of $\Omega_{k, 1}$.

Case II. $\alpha^{*}>p-n$. Again it is enough to deal with $\gamma=\left(a^{*}+n\right) p /\left(a^{*}+n-p\right)$. From Theorem 1.3.3

$$
\left\{\int_{\Omega_{k, 1}}|\nu(y)|^{\nu}\left[r_{k}(y)\right]^{\alpha} d y\right\}^{1 / \nu} \leq J_{1}\left\{\int_{\Omega_{k, 1}}\left[|\nu(y)|^{p}+|\nabla \nu(y)|^{p}\right]\left[r_{k}(y)\right]^{\alpha} d y\right\}^{1 / p}
$$

where $\nu=(\alpha+n) p /(\alpha+n-p) \geq \gamma$ and $J_{1}$ is independent of $\alpha$ for $p-n<a_{0} \leq$ $a \leq a^{*}$. (See Remark 4 following Theorem 1.3.3.) By Hölder's inequality and since $r_{k}(y) \leq 1$ in $\Omega_{k, 1}$ we have

$\left\{\int_{\Omega_{k, 1}}|\nu(y)|^{\gamma}\left[r_{k}(y)\right]^{\alpha} d y\right\}^{1 / \gamma} \leq\left\{\int_{\Omega_{k, 1}}|\nu(y)|^{\nu}\left[r_{k}(y)\right]^{\alpha} d y\right\}^{1 / \nu}\left[\operatorname{vol} \Omega_{k, 1}\right]^{(\nu-\gamma) / \nu \gamma}$

so that if $a_{0} \leq \alpha \leq a^{*}$ then (6) follows from (8).

If $p-n<0$ we can take $\alpha_{0}=0$ and be done. Otherwise $p \geq n \geq 2$. Fixing $a_{0}=\left(a^{*}-n+p\right) / 2$ we can find $0 \leq \alpha_{1}<p-n$ (or $\alpha_{1}=0$ if $p=n$ ) such that for $a_{1} \leq a \leq a_{0}$ we have

$$
1 \leq q \equiv \frac{(\alpha+n)\left(\alpha^{*}+n\right) p}{(\alpha+n)\left(\alpha^{*}+n\right)+\left(\alpha^{*}-\alpha\right) p} \leq \frac{p}{1+\epsilon_{0}}
$$

where $\epsilon_{0}>0$ depends only on $\alpha^{*}, n$ and $p$. Because of the latter inequality we may clearly also assume that $q-n<\alpha_{1}$. Since $(\alpha+n) q /(\alpha+n-q)=\gamma$ we have, 
again by Theorem 1.3 .3 and Hölder's inequality,

$$
\left\{\int_{\Omega_{k, 1}}|\nu(y)|^{\gamma}\left[r_{k}(y)\right]^{\alpha} d y\right\}^{1 / \gamma} \leq J_{2}\left\{\int_{\Omega_{k, 1}}\left[|\nu(y)|^{q}+|\nabla v(y)|^{q}\right]\left[r_{k}(y)\right]^{\alpha} d y\right\}^{1 / q}
$$

$$
\leq 2^{(p-q) / p q} J_{2}\left\{\int_{\Omega_{k, 1}}\left[|v(y)|^{p}+|\nabla v(y)|^{p}\right]\left[r_{k}(y)\right]^{\alpha} d y\right\}^{1 / p}\left[\operatorname{vol} \Omega_{k, 1}\right]^{(p-q) / p q}
$$

where $J_{2}$ is independent of $\alpha$ for $\alpha_{1} \leq a \leq \alpha_{0}$.

In case $\alpha_{1}>0$ we can obtain a similar (uniform) estimate for $0 \leq a \leq \alpha_{1}$ by the method of Case I. Combining this with (8) and (9) proves (6) for this case.

Case III. $\alpha^{*}=p-n, 1 \leq \gamma<\infty$. Fix $\nu \geq \max (\gamma, n /(n-1))$ and let $q=$ $(\alpha+n) \nu /(\alpha+n+\nu)$ so that $\nu=(\alpha+n) q /(\alpha+n-q)$. Then $1 \leq q \leq p \nu /(p+\nu)<p$ for $0 \leq a \leq \alpha^{*}$. Hence we can select $\alpha_{1} \geq 0$ such that $q-n<\alpha_{1}<p-n$. The rest of the proof is similar to that of Case II. This completes the lemma.

The above lemma indicates that for domains with power-like cusps we should expect some imbeddings of the form $W^{m, p}(\Omega) \rightarrow L^{q}(\Omega)(q>p)$. We generalize the lemma as follows.

2.3.3 Theorem. Let $\Omega$ be an open domain in $E_{n}$ baving the following property: there exists a family $\Gamma$ of open subsets of $\Omega$ such that

(i) $\Omega=\bigcup_{G \in \Gamma} G$;

(ii) $\Gamma$ has the finite intersection property, i.e. there exists a positive integer $N$ such that any $N+1$ distinct sets in $\Gamma$ have empty intersection;

(iii) at most one $G \in \Gamma$ bas the cone property;

(iv) there exist positive constants $\alpha^{*}>m p-n$ and $A$ such that for any $G \in \Gamma$ not having the cone property there exists a one-to-one function $\psi$ mapping $G$ onto a standard power cusp $\Omega_{k, \lambda}$ where $(\lambda-1) k=\alpha \leq \alpha^{*}$ and such that for all $i$ and $j$ $(1 \leq i, j \leq n)$, all $x \in G$ and all $y \in \Omega_{k, \lambda}$

$$
\left|\partial \psi_{j} / \partial x_{i}\right| \leq A, \quad\left|\partial\left(\psi^{-1}\right)_{j} / \partial y_{i}\right| \leq A
$$

If $p \leq \gamma \leq\left(\alpha^{*}+n\right) p /\left(\alpha^{*}+n-m p\right)$ then

$$
W^{m, p}(\Omega) \rightarrow L^{\gamma}(\Omega) .
$$

Proof. First note that it is sufficient to prove the theorem in the special case $m=1$, for, assuming this done, we have for $|s| \leq m-1, q=\left(\alpha^{*}+n\right) p /\left(\alpha^{*}+n-p\right)$, and $u \in W^{m, p}(\Omega)$

$$
\left|D^{s} u: L^{q}(\Omega)\right| \leq K_{3}\left|D^{s} u: W^{1, p}(\Omega)\right| \leq K_{4}\left|u: W^{m, p}(\Omega)\right|
$$

whence $\left|u: W^{m-1, q}(\Omega)\right| \leq K_{5}\left|u: W^{m, p}(\Omega)\right|$. Since 


$$
\frac{\left(a^{*}+n\right) q}{\alpha^{*}+n-(m-1) q}=\frac{\left(a^{*}+n\right) p}{\alpha^{*}+n-m p}
$$

the case of general $m$ follows by induction.

We assume now that $m=1$. Let $u \in C^{1}(\Omega)$ and let $G \in \Gamma$. If $G$ has the cone property then since $p \leq \gamma \leq\left(\alpha^{*}+n\right) p /\left(\alpha^{*}+n-p\right)<n p /(n-p)$ we have by Theorem 2.1.1

$$
\left|u: L^{\gamma}(G)\right| \leq K_{6}\left|u: W^{1, p}(G)\right|
$$

If $G$ does not have the cone property and $\psi: G \rightarrow \Omega_{k, \lambda}$ is as specified above, then by Lemma 2.3.2

$$
\begin{aligned}
\left|u: L^{\gamma}(G)\right| & \leq K_{7}\left|u \circ \psi^{-1}: L^{\gamma}\left(\Omega_{k, \lambda}\right)\right| \\
& \leq K_{8}\left|u \circ \psi^{-1}: W^{1, p}\left(\Omega_{k, \lambda}\right)\right| \leq K_{9}\left|u: W^{1, p}(G)\right|
\end{aligned}
$$

where $K_{9}$ is independent of $G \in \Gamma$. We have, therefore (noting that $p / \gamma \leq 1$ ),

$$
\begin{aligned}
\left|u: L^{\gamma}(\Omega)\right|^{p} & \leq\left\{\sum_{G \in \boldsymbol{\Gamma}} \int_{G}|u(x)|^{\gamma} d x\right\}^{p / \gamma} \\
& \leq \sum_{G \in \boldsymbol{\Gamma}}\left\{\int_{G}|u(x)|^{\gamma} d x\right\}^{p / \gamma} \\
& \leq K_{10} \sum_{G \in \Gamma} \int_{G}\left[|u(x)|^{p}+|\nabla u(x)|^{p}\right] d x \leq K_{10} N\left|u: W^{1, p}(\Omega)\right|^{p}
\end{aligned}
$$

where we have used the finite intersection property of $\Gamma$ in the last inequality. The imbedding (10) now follows by completion.

Remarks. 1. Imbeddings of the sort (10) for certain domains having cusps were established by I. Globenko ([5], [6]) by a different technique. The limiting exponent $\gamma=\left(\alpha^{*}+n\right) p /\left(\alpha^{*}+n-m p\right)$ is not covered by Globenko's method. See also Maz'ya ([8], [9]).

2. If $\alpha^{*} \leq m p-n$ then (10) holds for $p \leq \gamma<\infty$.

3. If (and only if) $\Omega$ has finite volume (10) holds in addition for $1 \leq \gamma<p$.

4. The theorem is best possible in the sense that if $(\lambda-1) k=\alpha>m p-n$ and $\gamma>(\alpha+n) p /(\alpha+n-m p)$ there exists a function $u$ in $W^{m, p}\left(\Omega_{k, \lambda}\right)$ but not in $L^{\gamma}\left(\Omega_{k, \lambda}\right)$. There are, of course, many domains having power-cusp-like singularities which, nevertheless, do not satisfy the conditions of Theorem 2.3.3. It is possible to generalize the methods of this paper to cover some of these.

Example. Let $\Omega=\left\{\left(x_{1}, x_{2}, x_{3}\right) \in E_{3}: x_{2}>0, x_{2}^{2}<x_{1}<3 x_{2}^{2}\right\}$. Setting $a=$ $[3 / 4 \pi]^{1 / 3}$ we may readily verify that the transformation

$$
y_{1}=x_{1}-2 x_{2}^{2}, \quad y_{2}=x_{2}, \quad y_{3}=x_{3}-k / a \quad(k=0,1,2, \ldots)
$$


transforms a subdomain $G_{k}$ of $\Omega$ onto the standard power cusp $\Omega_{1,2}$ in the manner required of $\psi$ in the statement of Theorem 2.3.3. Moreover $\left\{G_{k}\right\}_{k=-\infty}^{\infty}$ has the finite intersection property and covers $\Omega$ up to a set with the cone property. Hence $W^{m, p}(\Omega) \rightarrow L^{\gamma}(\Omega)$ for $p \leq \gamma \leq 4 p /(4-m p)$ if $m p<4$, or for $p \leq \gamma<\infty$ if $m p \geq 4$.

We now consider imbeddings into spaces of continuous functions.

2.3.4 Lemma. Let $0 \leq \alpha^{*}<m p-n$. There exists a constant $Q=Q\left(n, p, a^{*}\right)$ such that if $1 \leq k \leq n-1$ and $\lambda \geq 1$ satisfy $a=(\lambda-1) k \leq \alpha^{*}$ then for all $u \in C^{m}\left(\Omega_{k, \lambda}\right)$

$$
\sup _{x \in \Omega_{k, \lambda}}|u(x)| \leq Q\left|u: W^{m, p}\left(\Omega_{k, \lambda}\right)\right| .
$$

Proof. First suppose $m=1$. For $u \in C^{1}\left(\Omega_{k, \lambda}\right)$ we have by Theorem 1.4.2 and via the method of the first part of the proof of Lemma 2.3.2

$$
\begin{aligned}
\sup _{x \in \Omega_{k, \lambda}}|u(x)| & =\sup _{y \in \Omega_{k, 1}}|u(y)| \\
& \leq Q_{1}\left\{\int_{\Omega_{k, 1}}\left[|u(y)|^{p}+\left|\nabla_{u}(y)\right|^{p}\right]\left[r_{k}(y)\right]^{a} d y\right\}^{1 / p} \\
& \leq Q_{2}\left\{\int_{\Omega_{k, \lambda}}\left[|u(x)|^{p}+\left|\nabla_{u}(x)\right|^{p}\right] d x\right\}^{1 / p} .
\end{aligned}
$$

Since $r_{k}(y) \leq 1$ for $y \in \Omega_{k, 1}$ it is clear that $Q_{1}$ and hence $Q_{2}$ can be chosen independent of $k$ and $\lambda$ for $a \leq a^{*}$.

For arbitrary $m$ we have by Theorem $2.3 .3 w^{m, p}\left(\Omega_{k, \lambda}\right) \rightarrow W^{1, \gamma}\left(\Omega_{k, \lambda}\right)$ for some $\gamma>\alpha^{*}+n$ (specifically $\gamma=\left(\alpha^{*}+n\right) p /\left(\alpha^{*}+n-(m-1) p\right)>\alpha^{*}+n$ if $(m-1) p-n<a^{*}$, or any $\gamma>a^{*}+n$ otherwise). It then follows from (12) that

$$
\sup _{x \in \Omega_{k, \lambda}}|u(x)| \leq Q_{3}\left|u: W^{1, \gamma}\left(\Omega_{k, \lambda}\right)\right| \leq Q\left|u: W^{m, p}\left(\Omega_{k, \lambda}\right)\right| .
$$

2.3.5 Theorem. Let $\Omega$ be a domain in $E_{n}$ with the following property: there exist positive constants $\alpha^{*}<m p-n$ and $A$ such that for each $x \in \Omega$ there exists an open set $G$ with $x \in G \subset \Omega$ and a one-to-one mapping $\psi$ of $G$ onto a standard power cusp $\Omega_{k, \lambda}$ with $(\lambda-1) k=a \leq a^{*}$ and such that, for $|s| \leq m$, $1 \leq i, j \leq n$, and for all $x \in G$ and $y \in \Omega_{k, \lambda}$

$$
\left|\partial \psi_{i} / \partial x_{j}\right| \leq \Lambda, \quad\left|D^{s}\left(\psi^{-1}\right)(y)\right| \leq A .
$$


Then

$$
W^{m, p}(\Omega) \rightarrow C(\bar{\Omega}) .
$$

More generally, if $\alpha^{*}<(m-j) p-n$ where $0 \leq j \leq m-1$ then

$$
W^{m, p}(\Omega) \rightarrow C^{j}(\bar{\Omega}) .
$$

Proof. It is sufficient to prove (13). If $\psi$ maps $G \subset \Omega$ onto $\Omega_{k, \lambda}$ we have for $u \in C^{m}(\Omega)$

$$
\begin{aligned}
\sup _{x \in G}|u(x)| & =\sup _{y \in \Omega_{k, \lambda}}\left|u \circ \psi^{-1}(y)\right| \\
& \leq Q_{3}\left|u \circ \psi^{-1}: W^{m, p}\left(\Omega_{k, \lambda}\right)\right| \leq Q_{4}\left|u: W^{m, p}(G)\right| .
\end{aligned}
$$

Since $Q_{4}$ is independent of $G$ we obtain

$$
\sup _{x \in \Omega}|u(x)| \leq Q_{4}\left|u: W^{m, p}(\Omega)\right| .
$$

By completion (15) holds for all $u \in W^{m, p}(\Omega)$. For such $u$ there exists a sequence $u_{n} \in C(\Omega)$ such that $u_{n} \rightarrow u$ in $W^{m, p}(\Omega)$. By (15) $u_{n}(x) \rightarrow u(x)$ uniformly almost everywhere in $\Omega$, whence $u \in C(\bar{\Omega})$ after possible redefinition on a set of measure zero.

2.3.6 Theorem. Let $\Omega$ be a domain in $E_{n}$ with the following property: there exist positive constants $\alpha^{*}, \delta$ and $A$ such that for each pair of points $x, y \in \Omega$ with $|x-y| \leq \delta$ there exists an open set $G$ with $x, y \in G \subset \Omega$ and a one-to-one mapping $\psi$ of $G$ onto some standard power cusp $\Omega_{k, \lambda}$ with $(\lambda-1) k=\alpha \leq \alpha^{*}$ and such that, for $|s| \leq m, 0 \leq i, j \leq n$, and for all $x \in G$ and $z \in \Omega_{k, \lambda}$ we bave

$$
\left|\partial \psi_{i} / \partial x_{j}\right| \leq A, \quad\left|D^{s}\left(\psi^{-1}\right)_{i}(z)\right| \leq A .
$$

Suppose that for some $l$ with $0 \leq l \leq m-1$ we have $(m-l-1) p<\alpha^{*}+n<$ $(m-l) p$ then $W^{m, p}(\Omega) \rightarrow C^{l, \mu}(\bar{\Omega}), 0<\mu \leq\left((m-l) p-n-\alpha^{*}\right) / p$. If $(m-l-1) p=$ $\alpha^{*}+n$ then $W^{m, p}(\Omega) \rightarrow C^{l, \mu}(\bar{\Omega}), 0<\mu<1$.

The details of the proof are similar to those of Lemma 2.3.4 and Theorem 2.3.5. We omit them.

We conclude by showing that the entire imbedding Theorem 2.1 .1 fails if $\Omega$ has cusps sharper than any power cusp. (Let us call such cusps exponential cusps.) Let $B_{r}=B_{r}\left(x_{0}\right)$ denote the ball of radius $r$ about $x_{0} \in E_{n} ; \Omega_{r}=B_{r} \cap \Omega$ and $S_{r}(\Omega)=\left(\partial B_{r}\right) \cap \Omega$. Let $A(r, \Omega)$ be the surface area (Lebesgue $(n-1)$-measure) of $S_{r}(\Omega)$. We shall say that $\Omega$ has an exponential cusp at $x_{0} \in \partial \Omega$ if for every real number $k$ we have 


$$
\lim _{r \rightarrow 0^{+}} A(r, \Omega) / r^{k}=0
$$

2.3.7 Theorem. If $\Omega$ is a domain in $E_{n}$ baving an exponential cusp at $x_{0} \epsilon$ $\partial \Omega$ there can be no imbedding of $W^{m, p}(\Omega)$ into $L^{q}(\Omega)$ for any $q>p$, or into $C^{j}(\bar{\Omega})$ for any $j$.

Proof. We construct a function $u \in W^{m, p}(\Omega)$ which fails to belong to $L^{q}(\Omega)$ $(q>p)$ or $C^{j}(\bar{\Omega})$ because it becomes unbounded too rapidly near $x_{0}$. We make use of Theorem 2.2.1 in the construction.

Without loss of generality we assume $x_{0}=0$ so that $r=|x|$. Let $\Omega^{*}=$ $\left\{y=x /|x|^{2}: x \in \Omega,|x|<1\right\}$. It is easily seen that $\Omega^{*}$ is unbounded and has finite volume, and that $A\left(r, \Omega^{*}\right)=r^{2(n-1)} A(1 / r, \Omega)$. Let $t$ satisfy $p<t<q$. By Theorem 2.2.1 there exists a function $\tilde{v} \in C^{m}(0, \infty)$ such that

(i) $\widetilde{v}(r)=0$ if $0<r \leq 1$,

(ii) $\int_{1}^{\infty}\left|\widetilde{v}^{(j)}(r)\right|^{t} A\left(r, \Omega^{*}\right) d r<\infty$ if $j=0,1, \ldots, m$,

(iii) $\int_{1}^{\infty}|\widetilde{v}(r)|^{q} A\left(r, \Omega^{*}\right) d r=\infty$.

(If $r=|y|$ then $v(y)=\widetilde{v}(r)$ defines $v \in W^{m, p}\left(\Omega^{*}\right)$ but $v \notin L^{q}\left(\Omega^{*}\right)$.) Let $x=$ $y /|y|^{2}$ so that $\rho=|x|=1 /|y|=1 / r$. Set $\beta=2 n / q$ and define $u(x)=\widetilde{u}(\rho)=$ ${ }_{r} \beta \tilde{v}(r)=|y|^{\beta} v(y)$. It follows for $|s|=j \leq m$ that

$$
\left|D^{s} u(x)\right| \leq\left|\widetilde{u}^{(j)}(\rho)\right| \leq \sum_{i=0}^{j} c_{i j} r^{\beta+j+i} \widetilde{v}^{(i)}(r)
$$

where $c_{i j}$ depends only on $\beta$. Now $u(x)$ vanishes for $|x| \geq 1$ and so

$$
\begin{aligned}
\int_{\Omega}|u(x)|^{q} d x & =\int_{0}^{1}|\tilde{u}(\rho)|^{q} A(\rho, \Omega) d \rho \\
& =\int_{1}^{\infty}|\widetilde{v}(r)|^{q} A\left(r, \Omega^{*}\right) d r=\infty .
\end{aligned}
$$

On the other hand, if $0 \leq|s|=j \leq m$,

$$
\begin{aligned}
\int_{\Omega}\left|D^{s} u(x)\right|^{p} d x & \leq \int_{0}^{1}\left|\tilde{u}^{(j)}(\rho)\right|^{p} A(\rho, \Omega) d \rho \\
& \leq \text { const } \sum_{i=0}^{j} \int_{1}^{\infty}\left|\tilde{v}^{(i)}(r)\right|^{p} r^{(\beta+j+i) p-2 n} A\left(r, \Omega^{*}\right) d r .
\end{aligned}
$$

If it happens that $(\beta+2 m) p \leq 2 n$ then, since $p<t$ and vol $\Omega^{*}<\infty$, we have by Hölder's inequality that all the integrals in (17) are finite and $u \in W^{m, p}(\Omega)$ completing the proof. Otherwise let

$$
k=[(\beta+2 m) p-2 n][t /(t-p)]+2 n .
$$

By (16) there exists $a \leq 1$ such that if $\rho \leq a$ then $\Lambda(\rho, \Omega) \leq \rho^{k}$. It follows that 
if $r \geq 1 / a$ then $r^{k-2 n} A\left(r, \Omega^{*}\right) \leq r^{k-2} \rho^{k}=r^{-2}$. Thus

$$
\begin{aligned}
\int_{1}^{\infty} & r^{(\beta+j+i) p-2 n}\left|\widetilde{v}^{(i)}(r)\right|^{p} A\left(r, \Omega^{*}\right) d r \\
& =\int_{1}^{\infty}\left|\widetilde{v}^{(i)}(r)\right|^{p} r^{(k-2 n)(t-p) / t} A\left(r, \Omega^{*}\right) d r \\
& \leq\left\{\int_{1}^{\infty}\left|\widetilde{v}^{(i)}(r)\right|^{t} A\left(r, \Omega^{*}\right) d r\right\}^{p / t}\left\{\int_{1}^{\infty} r^{k-2 n} A\left(r, \Omega^{*}\right) d r\right\}^{(t-p) / t}
\end{aligned}
$$

whence $u \in W^{m, p}(\Omega)$ and the proof is complete.

\section{REFERENCES}

1. R. A. Adams and John Fournier, Some imbedding theorems for Sobolev spaces, Canad. J. Math. 23 (1971), 517-530.

2. Rolf Andersson, Unbounded Soboleff regions, Math. Scand. 13 (1963), 75-89. MR $31 \# 3847$.

3. C. W. Clark, Introduction to Sobolev spaces, Seminar Notes, University of British Columbia, Vancouver, 1968.

4. E. Gagliardo, Proprietà di alcune classi di funzioni in più variabili, Ricerche Mat. 7 (1958), 102-137. MR 21 \#1526.

5. I. G. Globenko, Embedding theorems for a region with null salient points, Dokl. Akad. Nauk SSSR 132 (1960), 251-253 = Soviet Math. Dokl. 1 (1960), 517-519. MR 24 \#A328.

6. - Some questions in the theory of imbedding for domains with singularities on the boundary, Mat. Sb. 57 (99) (1962), 201-224. MR $26 \# 589$.

7. G. Hellwig, Differential operators of mathematical physics. An introduction, Springer-Verlag, Berlin and New York, 1964; English transl., Addison-Wesley, Reading, Mass., 1967. MR 29\#2682; MR 35\#2174。

8. V. G. Maz'ja, Classes of domains and imbedding theorems for function spaces, Dokl. Akad. Nauk SSSR 133 (1960), 527-530 = Soviet Math. Dokl. 1 (1960), 882-885. MR 23 \#A3448.

9. - $p$-conductance and theorems of imbedding certain function spaces into the space C, Dokl. Akad. Nauk SSSR 140 (1961), 299-302 = Soviet Math. Dokl. 2 (1961), 1200-1203. MR $28 \# 460$.

10. N. G. Meyers and J. Serrin, $H=W$, Proc. Nat. Acad. Sci. U.S. A. 51 (1964), 1055-1056. MR 29\#1551.

11. C. B. Morrey, Jr., Functions of several variables and absolute continuity. II, Duke Math. J. 6 (1940), 187-215. MR 1, 209.

12. S. L. Sobolev, Applications of functional analysis in mathematical physics, Izdat. Leningrad. Gos. Univ., Leningrad, 1950; English transl., Transl. Math. Monographs, vol. 7, Amer. Math. Soc., Providence, R. I., 1963. MR 14, 565; MR 29 \#2624.

13. N. S. Trudinger, On imbeddings into Orlicz spaces and some applications, J. Math. Mech. 17 (1967), 473-483. MR 35 \#7121.

14. A. Zygmund, Trigonometrical scries. Vol. 1, 2nd ed., Cambridge Univ. Press, New York, 1959. MR $21 \# 6498$.

DEPARTMENT OF MATHEMATICS, UNIVERSITY OF BRITISH COLUMBIA, VANCOUVER, BRITISH COLUMBIA, CANADA 